\title{
Visibilidad e impacto de las revistas peruanas de Ciencias Sociales en acceso abierto
}

\author{
Luís Fernando Morales Morante \\ Universidad Autónoma de Barcelona - UAB, España
}

ARTICLE

\begin{abstract}
Resumen
En el presente artículo se analiza la producción científica y los principales indicadores de visibilidad internacional de 24 revistas peruanas de Ciencias Sociales indexadas en el Catálogo Latindex entre los años 2005-2014, disponibles en acceso abierto. Los resultados indican un mayor número de revistas del campo de la Psicología, Ade-Economía y Derecho, respecto del resto de áreas de conocimiento. Se observa un volumen importante de artículos publicados, así como un predominio de artículos escritos por autores nacionales sobre los extranjeros. Se constata un claro dominio de las universidades privadas, respecto de las públicas en la tarea editorial de revistas científicas. Se proponen acciones e iniciativas de incentivo para mejorar el impacto, calidad, número de citas y la presencia en motores de búsqueda internacional.
\end{abstract}

Palabras clave

Visibilidad ; Impacto ; Ciencias Sociales ; Revistas científicas ; Acceso abierto ; Latindex ; Perú

Visibility and impact of peruvian social science journals in open access

\begin{abstract}
In this article the scientific production and leading indicators of international visibility of 24 Peruvian social science journals indexed in the Catalog Latindex between 2005-2014, available in Open Access is analyzed. The results indicate a greater number of magazines in the field of psychology, Ade-Economics and Law, with respect to other areas of knowledge. a significant volume of published articles and a predominance of articles written by local authors on foreigners is observed. a clear dominance of private universities, from public task in the publishing of scientific journals is found. incentive measures and initiatives are proposed to improve the impact, quality, number of citations and presence in international search engines.
\end{abstract}

Keywords

Visibility ; Impact ; Social Sciences ; Journals ; Open access; Latindex ; Peru

\section{Introducción}

El Perú viene destacando como una de las economías lideres en América Latina. Entre 2004 y 2015 alcanzó una tasa de crecimiento del Producto Interno Bruto (PIB) de 6,1\% (Banco Mundial, 2015). En el campo de la educación, dicho crecimiento se viene reflejando en la creación de un gran número de universidades y centros superiores de estudio. Según datos de la Asamblea Nacional de Rectores (ANR), órgano supervisor del sistema universitario peruano y reportados a la consultora internacional Microconsult, en solo 13 años el número de universidades en el Perú prácticamente se duplicó y se pasó de 72 a 140. La cantidad de universidades 
públicas creció en 59\%, mientras que las privadas lo hicieron a 123\% (Diario Gestión, 2014). En este contexto, las carreras de Ciencias Sociales vienen siendo las más demandadas de la actual oferta educativa nacional. En 2014, la carrera de Derecho registró un total de 57,915 alumnos, seguida de Contabilidad con 55,317 y en tercer lugar Administración con 48,512 (Expo Universidad, 2014). Como consecuencia de este crecimiento desbordado se viene observando una feroz competencia entre universidades por conseguir una parcela de mercado que permita su consolidación para los próximos años. Una forma de conseguir diferenciarse de sus competidores ha sido a través de certificaciones privadas internacionales que han validado tanto carreras, facultades como incluso universidades enteras. Por ejemplo, en el año 2010 la facultad de Derecho de la Universidad de San Martín de Porres fue acreditada por la (AQ) Agencia Catalana de la Calidad (USMP, 2010). La Universidad Continental se acreditó en 2015 por la Agencia Nacional de Evaluación y Calidad Académica (Aneca) (Continental, 2015), mientras que la Facultad de Comunicaciones de la Universidad de Ciencias Aplicadas (UPC) obtuvo en 2014 la acreditación del Consejo Latinoamericano de Acreditación de la Educación en Periodismo (CLAEP), por citar solo algunos casos conocidos. En medio de este escenario, surge la nueva Ley Universitaria (Diario Oficial El Peruano, 2014), que dedica un capítulo especial a la acreditación y certificación de las titulaciones. En la nueva norma se obliga a potenciar la investigación en los centros, a través de la formación de grupos de trabajo estables, competitivos y que publiquen en revistas de impacto. Asimismo, para obtener resultados favorables en la evaluación, la ley recomienda que los centros editen revistas de alcance internacional, como incentivo para que los docentes investiguen y publiquen el resultado de sus trabajos en revistas de reconocido prestigio internacional (Miyahira, 2011). Con miras a estos futuros procesos, es importante que los investigadores, editores de las revistas y las autoridades universitarias, conozcan cuál es el efecto de sus decisiones y actuaciones respecto de las publicaciones que gestionan. Y sobretodo, deben conocer cuáles son sus fortalezas y debilidades para que sus revistas, en un corto o mediano plazo, alcancen un nivel suficiente para ser incorporadas no solo en los catálogos de ámbito lberoamericano sino mundial como JCR o Scopus.

\section{Estado de la cuestión}

Desde hace quince años ha comenzado a desarrollarse una línea centrada en analizar los parámetros de calidad y visibilidad de las revistas editadas en Perú, concretamente en el campo de la medicina y las biociencias. Burstein y Cabezas (2010) analizan la rigurosidad de los procesos de presentacion de originales y del arbitraje internacional, como factores que garantizan la calidad editorial de las publicaciones. Romaní y Wong (2009) elaboran un estudio bibliometrico de articulos consignados en el catálogo SciELO. Los resultados detectan un predominio de estudios de caso que suponen menor rigor metodologico que las investigaciones científicas.

En el Perú, en el ámbito de las Ciencias Sociales, no existen antecedentes de investigación bibliométrica, cuantitativa o cualitativa que analice la producción global o el impacto de las publicaciones científicas. La juventud de muchas facultades, dedicadas principalmente a la docencia de pregrado, ha hecho que los profesores, en su mayoría sin grado de magíster o doctor y con bajos salarios, no vean en la investigación una opción atractiva para desarrollar su carrera profesional en la universidad. Según datos proporcionados por la ANR, en las universidades privadas el $66 \%$ de los profesores tienen dedicación solo a tiempo parcial y la universidad con más docentes doctores es la Pontificia Universidad Católica del Perú con solo un $21 \%$ de su plantilla (Expo Universidad, 2014). Las universidades más antiguas suelen poseer planas docentes consolidadas y experiencia en investigación. Poseen grupos estables, desarrollan líneas de estudio y consecuentemente están acostumbradas a publicar regularmente en los circuitos académicos internacionales. Sin embargo, esta práctica no suele suceder en las universidades mas jóvenes, en especial las privadas, cuyo nivel académico suele ser en general más bajo. La propia estructura del sistema universitario vigente no estimula la creación de nuevo conocimiento. No hay interés por desarrollar proyectos que se complementen con su actividad docente. No parece existir una articulación definida de su actividad formativa con los mecanismos para producir y dar a conocer el trabajo intelectual. El Concejo Nacional de Ciencia y Tecnología, órgano responsable de la investigación en el Perú, se limita a convocar ayudas dirigidas a las ciencias exactas y médicas, como sucede con el programa Cienciactiva (Concytec, 2016). Otro factor que dificulta la investigación en el ámbito específico de las Ciencias Sociales, es la abundancia de paradigmas mixtos para el abordaje de 
fenómenos complejos y muchas veces altamente cambiantes. Existe un exceso de teorías y modelos conceptuales generales o en proceso de elaboración y una suerte de desconexion que impide convertir dichos estudios en artículos apropiados para el formato que exigen las revistas científicas. La suma de todos estos factores, desde nuestro punto de vista, puede verse reflejada en un volumen desigual de producción de artículos y una limitada diversidad temática y de autores que traen finalmente como consecuencia una escasa visibilidad e impacto internacional de las revistas editadas en el Perú.

En España hay algunos trabajos interesantes que vienen estudiando la calidad de las revistas y sugieren una serie de indicadores fiables para medir la calidad de las revistas en el ámbito de las Ciencias Sociales. Consideramos que estos trabajos son pertinentes para extrapolarlos al contexto peruano y abordar nuestro problema de investigación. En esta línea, Román y Giménez, proponen un modelo de evaluacion especializado en revistas economicas basado en criterios de calidad formal, indicadores indirectos de calidad y juicio de los «pares» en la plataforma española INRECS (Román y Giménez, 2000). Borrego y Urbano (2006) examinan las dificultades de aplicación, en estas disciplinas, de métodos bibliométricos de repercusión basados en el análisis de citas con datos obtenidos de las bases de datos de Thomson Scientific (ex ISI). Más recientemente, Fernández Quijada y otros, analizan la calidad de las revistas españolas de Comunicación, a partir del volumen de articulos, el cálculo y procedencia de autores y citaciones internacionales (Fernández-Quijada y otros, 2013; Fernández Quijada, 2010). Tambien se constatam los posibles efectos negativos de las auto-citas en el factor de impacto (Campanario y Candelario, 2010), o la transparencia de parametros informativos declarados por las propias revistas (Tavares, 2011). Para incrementar el impacto de las publicaciones en acceso abierto, TorresSalinas y otros (2009), sugieren un conjunto de estrategias para mejorar el impacto y visibilidad de las publicaciones a traves de herramientas en Red 2.0. Algunos estudios también se han realizado en el Brasil y recientemente en Perú. Shintaku y otros (2014), analizan las revistas OA y discuten los escollos que deben superar las publicaciones cientificas para su alojamiento en portales institucionales. Constata la necesidad de mayores esfuerzos para lograr ser incroporadas en el catalogo SciELO. Morales (2015), examina precisamente las revistas de Ciencias Sociales en Perú y detecta una alta concentración de autores de los propios centros que publican la revista y un reducido porcentaje de autores extranjeros. También advierte una elevada discontinuidad en la publicación de los números y demora en los procesos de revisión peer review, entre otros. Se hace necesario, primeramente, obtener información más completa y precisa de la cantidad y calidad de los artículos publicados para poder analizar las posibles variaciones en la productividad de las revistas y encontrar las causas para fortalecer su posicionamiento en los próximos años.

Actualmente el movimiento Open Access (OA) representa una vía bastante extendida para mejorar la visibilidad internacional y citaciones de las revistas (Sánchez, 2007). En el Perú la gran mayoría de revistas académicas son accesibles en OA, aunque todavía muchas de ellas siguen editándose simultáneamente en versión papel (Laakso y otros, 2011). Si bien es verdad, que el solo hecho que una revista sea publicada en OA no necesariamente implica calidad o sea un factor para que tenga más lecturas y citaciones, si que las buenas prácticas de gestión y difusión hechas por los editores, pueden contribuir a ello en varios sentidos: incrementar el volumen de autores, diversificar la procedencia de contribuciones, aumentar el número de consultas o descargas. Tambíen puede contribuir a mejorar la periodicidad y control de los procedimientos editoriales. El cumplimento de estos criterios de calidad, son fundamentales para que las publicaciones sean finalmente incluidas en buscadores y catálogos de revistas internacionales.

En nuestra revisión de repositorios y portales se ha podido constatar que muchas revistas peruanas del campo de las Ciencias Sociales no aparecen en motores de referencia iberoamericana como SciELO, Redalyc, Clase o incluso Dialnet. Para Barreto De Castro (2005), este hecho refleja la incapacidad de construir un modelo propio y plantea una larga labor coordinada para los próximos años. En Perú, el motor SciELO (Perú), coordinado por el Consejo Nacional de Ciencia, Tecnología e Innovación Tecnológica (Concytec), la Universidad Nacional Mayor de San Marcos (UNMSM) y la Representación en Perú de la Organización Panamericana de la Salud (OPS), registra únicamente cinco revistas de Ciencias Sociales y 38 volúmenes disponibles. Aun cuando varios indicadores bibliométricos no se encuentren disponibles en su totalidad. Redalyc incluye 6 revistas de Ciencias Sociales en su buscador. El Concytec ha creado el Repositorio Alicia (Acceso Libre a la Información Científica) como una iniciativa interesante para aglutinar la producción científica nacional. El sistema dispone al acceso abierto de artículos y tesis, búsquedas avanzadas por temas, que enlazan al usuario con la web oficial de la revista, pero no ofrecen datos de impacto de revistas ni citaciones de artículos. Ante estas carencias, en algunos campos como la Economía, los editores de las revistas prefieren destinar sus esfuerzos a incluirlas en 
plataformas especiales de su área como LogEC o Ideas, que ofrecen otros datos no disponibles en los buscadores antes referidos. Esto disgrega todavía más no solo la búsqueda general de revistas sino el acceso a indicadores que puedan ser luego comparables con otras revistas. Consideramos entonces que hace falta un estudio actual y completo que brinde información bibliométrica avanzada y comparada de la producción y visibilidad de las revistas peruanas del ámbito de las Ciencias Sociales.

\section{Objetivos}

Ante la falta de un repositorio nacional que ofrezca datos completos de la producción científica del país, se hace necesario iniciar la labor con un levantamiento de datos a nivel nacional, con información detallada del material publicado por cada revista y sus caracteristicas, con el fin de disponer de diferentes indicadores de impacto, autores, procedencia de las contribuciones, coautorías, visibilidad y repercusion internacional. Este trabajo puede ser importante de cara a la mejora de la gestión ordinaria de las publicaciones. Es muy oportuno también por la coyuntura actual de crecimiento y fase de ordenamiento del sistema universitario peruano. Hacia la consecución de estos dos propósitos en esta investigación se formulan los siguientes objetivos:

\subsection{Objetivo general:}

- Efectuar un estudio cuantitativo y cualitativo de la producción científica de las revistas peruanas del ámbito de las Ciencias Sociales en versión digital abierta entre los años 2005 y 2014, a partir de indicadores estandarizados de visibilidad e impacto.

El rango de tiempo de diez años se establece para considerar en el análisis las nuevas publicaciones del ámbito aparecidas junto con la creación de facultades y centros de estudios unversitarios.

\subsection{Objetivos específicos:}

- Analizar, a partir de los datos, el nivel de posicionamiento de las revistas e instituciones que las producen en el contexto nacional e internacional.

- Proponer medidas orientadas a mejorar la calidad y el nivel de impacto internacional de las publicaciones.

Finalmente, a los investigadores, este estudio puede ayudarles a saber mejor dónde deben publicar para mejorar el impacto de su obra y articular redes de trabajo en el exterior que permitan el acceso a nuevos circuitos para generar conocimiento especializado.

\section{Metodología y fuentes}

En este estudio lleva a cabo un recuento bibliométrico de los artículos publicados en revistas científicas entre los años 2005 y $2014 \mathbf{i}$.

El proceso de investigación se desarrolla en cuatro fases:

- Primera fase: Localización de la totalidad de revistas indexadas publicadas en Perú del ámbito de Ciencias Sociales en el Catálogo Latíndexii. Para efectuarla se ingresa al buscador avanzado de Latíndex/ Tema: Ciencias Sociales/ Indizada-Resumida en: Latíndex Catálogo/ País: Perú. 
- Segunda fase: Inventario de la totalidad de artículos publicados por cada revista entre los años 2005-2014. La recogida de datos se realiza manualmente desde la página web oficial de cada una de las publicaciones. La información del inventario individual se complementa con otros indicadores bibliométricos y de calidad disponibles en los buscadores SciELO-Perú, Clase y Redalyc, debido a que Latindex Catálogo no ofrece información de autores, países, citas o factor de impacto.

- Tercera fase: Construcción de una base de datos de los artículos científicos, número y procedencia de autores, periodicidad, indizaciones y otros criterios de calidad, recogidos del los propios portales u otros motores especializados.

- Cuarta fase: Análisis comparativo organizado de los datos, distribuido en 3 campos y 14 variables.

\subsection{Indicadores del análisis}

La elección de las variables de análisis se realiza con el objetivo de obtener información de las publicaciones en 3 campos (Tabla 1):

- Primero: Datos generales: Indican el número de revistas, especialidad temática, institución que la edita y frecuencia de publicación.

- Segundo: Producción Científica: Este campo persigue cuantificar el volúmen y distribución de artículos y la organización de las autorías en cada revista.

- Tercero: Otros indicadores de calidad internacional: Incluye la visibilidad de la revista en otros motores, visitas a los websites, número de citaciones en SciELO, Google Académico, CLASE, número de criterios Latíndex cumplidos, visitas al portal y descargas de artículos.

Tabla 1: Indicadores de análisis

\begin{tabular}{|c|c|c|c|}
\hline Campos & & Variables & Descriptor \\
\hline \multirow{5}{*}{ Datos generales } & 1 & Número de revistas & \multirow{5}{*}{$\begin{array}{l}\text { Indica la distribución de la producción } \\
\text { editorialde las revistas según } \\
\text { especialidades, centros } \\
\text { y periodicidad de publicación }\end{array}$} \\
\hline & 2 & Área de especialidad & \\
\hline & 3 & Institución & \\
\hline & 5 & Naturaleza de la institución & \\
\hline & 5 & Frecuencia de la publicación & \\
\hline \multirow{3}{*}{$\begin{array}{l}\text { Producción } \\
\text { Científica }\end{array}$} & 1 & Artículos publicados & \multirow{3}{*}{$\begin{array}{l}\text { Muestra el volumen de artículos, la } \\
\text { distribución } \\
\text { de autores nacionales y extranjeros. }\end{array}$} \\
\hline & 2 & Autores nacionales & \\
\hline & 3 & Autores extranjeros & \\
\hline \multirow{8}{*}{$\begin{array}{l}\text { Otros } \\
\text { Indicadores } \\
\text { de calidad } \\
\text { internacional }\end{array}$} & 1 & Indexación en otros motores & \multirow{8}{*}{$\begin{array}{l}\text { Presencia en otros motores, citaciones en } \\
\text { Google Académico, Scielo Clase y criterios } \\
\text { Latindex Cumplidos } \\
\text { Otros indicadores de consulta de portales y } \\
\text { lectores potenciales en los artículos. }\end{array}$} \\
\hline & 2 & Número de citaciones GA & \\
\hline & 3 & Número de citaciones SciELO & \\
\hline & 4 & Factor de impacto SciELO & \\
\hline & 5 & Artículos en CLASE & \\
\hline & 6 & Criterios Latindex cumplidos & \\
\hline & 7 & Visitas al portal web & \\
\hline & 8 & Descargas de artículos & \\
\hline
\end{tabular}

\section{Resultados}

El resultado del buscador muestra un total de 58 títulos de revistas, que sin discriminación inicial se van analizando individualmente y sus correspondientes datos se ingresan en un fichero especial en formato .xls. Descartamos del inventario las revistas equivocadamente catalogadas en el ámbito de Ciencias Sociales, suprimimos aquellas no disponibles en Acceso Abierto y finalmente las que no superan 5 años o números de discontinuidad, de los 10 años elegidos para el estudio. Este intervalo se considera razonable para que no afecte significativamente una lectura lineal y comparativa del total a analizar. Además, porque dicha ausencia puede obedecer a una interrupción involuntaria o un simple retraso de la publicación de la revista en versión 
digital. Finalmente, luego del filtraje se obtiene un listado de 24 revistas, con los siguientes resultados generales (ver Tabla 2).

Tabla 2: Datos generales

\begin{tabular}{|c|c|c|c|c|c|}
\hline & REVISTA & Área & Institución & Nat. Inst. & Frecuencia \\
\hline 1 & Anthropológica & Arq/Antr/Hist & U. Católica & U. Privada & Anual \\
\hline 2 & Apuntes & ADE-Economía & U. Pacífico & U. Privada & Semestral \\
\hline 3 & Boletín de arqueología & Arq/Antr/Hist & U. Católica & U. Privada & Anual \\
\hline 4 & Comunic@acción & Comunicación & U. Altiplano & U. Pública & Anual \\
\hline 5 & Contabilidad y negocios & ADE-Economía & U. Católica & U. Privada & Semestral \\
\hline 6 & Contratexto & Comunicación & U. de Lima & U. Privada & Anual \\
\hline 7 & CD J.of economics & ADE-Economía & E. Negocios & U. Privada & Semestral \\
\hline 8 & Debates de sociología & Ciencias Sociales & U. Católica & U. Privada & Anual \\
\hline 9 & Derecho PUCP & Derecho & U. Católica & U. Privada & Anual \\
\hline 10 & Economía & ADE-Economía & U. Católica & U. Privada & Anual \\
\hline 11 & Educación & Educación & U. Católica & U. Privada & Anual \\
\hline 12 & Histórica & Arq/Antr/Hist & U. Católica & U. Privada & Semestral \\
\hline 13 & Investigación educativa & Educación & U. San Marcos & U. Publica & Semestral \\
\hline 14 & Investigaciones sociales & Ciencias Sociales & U. San Marcos & U. Publica & Semestral \\
\hline 15 & J. Centrum cathedra & ADE-Economía & U. Católica & U. Privada & Semestral \\
\hline 16 & Liberabit & Psicología & U. San Martín & U. Privada & Semestral \\
\hline 17 & Persona & Psicología & U. de Lima & U. Privada & Anual \\
\hline 18 & Quipukamayoc & ADE-Economía & U. San Marcos & U. Publica & Semestral \\
\hline 19 & R. Comunicación & Comunicación & U. de Piura & U. Privada & Anual \\
\hline 20 & R. Derecho & Derecho & U. de Piura & U. Privada & Anual \\
\hline 21 & R. Inv. Psicología & Psicología & U. San Marcos & U. Publica & Semestral \\
\hline 22 & R. Comp. Int. & Derecho & Indecopi & Institución & Semestral \\
\hline 23 & Revista de Psic. PUCP & Psicología & U. Católica & U. Privada & Anual \\
\hline 24 & Revista de Psic. UCV & Psicología & U. C. Vallejo & U. Privada & Semestral \\
\hline
\end{tabular}

\subsection{Campo 1: Datos generales:}

\subsubsection{Número de revistas: 24}

5.1.2 Áreas de especialidad: Visualizando el gráfico inferior (Gráfico 1), apreciamos que la mayor concentración se produce en el área de Ade/Economía con un total de 6 revistas. Le sigue Psicología con 5 . Con 3 Arqueología/Antropología/Historia, Comunicación y Derecho. Más relegadas, con 2, Educación y Ciencias Sociales (general).

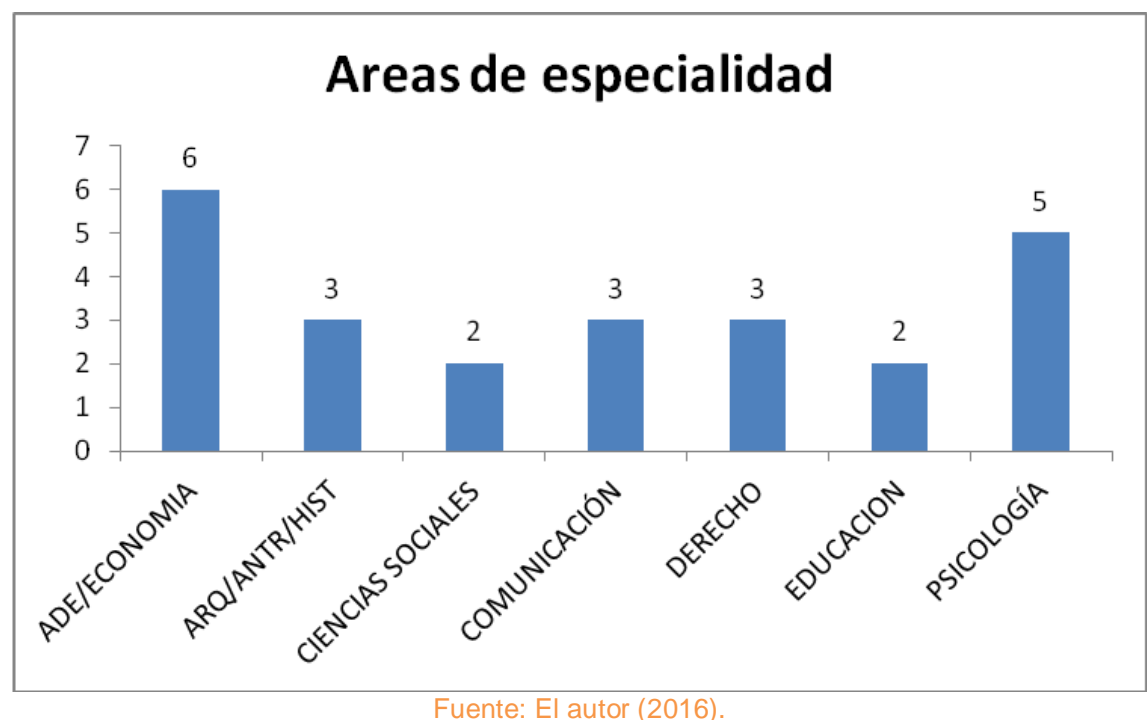


5.1.3 Institución: A través de esta variale podemos observar la distribución editorial según instituciones (ver Gráfico 2). El centro con mayor número de revistas es la Pontificia Universidad Católica del Perú (PUCP) con 10 revistas. Luego bastante postergado con 4 la Universidad Nacional Mayor de San Marcos (UNMSM). Con 2 la Universidad de Lima (UL) y la Universidad de Piura (UDEP), también privadas. El resto de centros solo edita una publicación: Esan, Indecopi, Universidad César Vallejo (UCV), Universidad Nacional del Altiplano (UNAL), Universidad del Pacífico (UPAC) y Universidad San Martín de Porres (USMP).

\section{Gráfico 2: Distribución por instituciones}

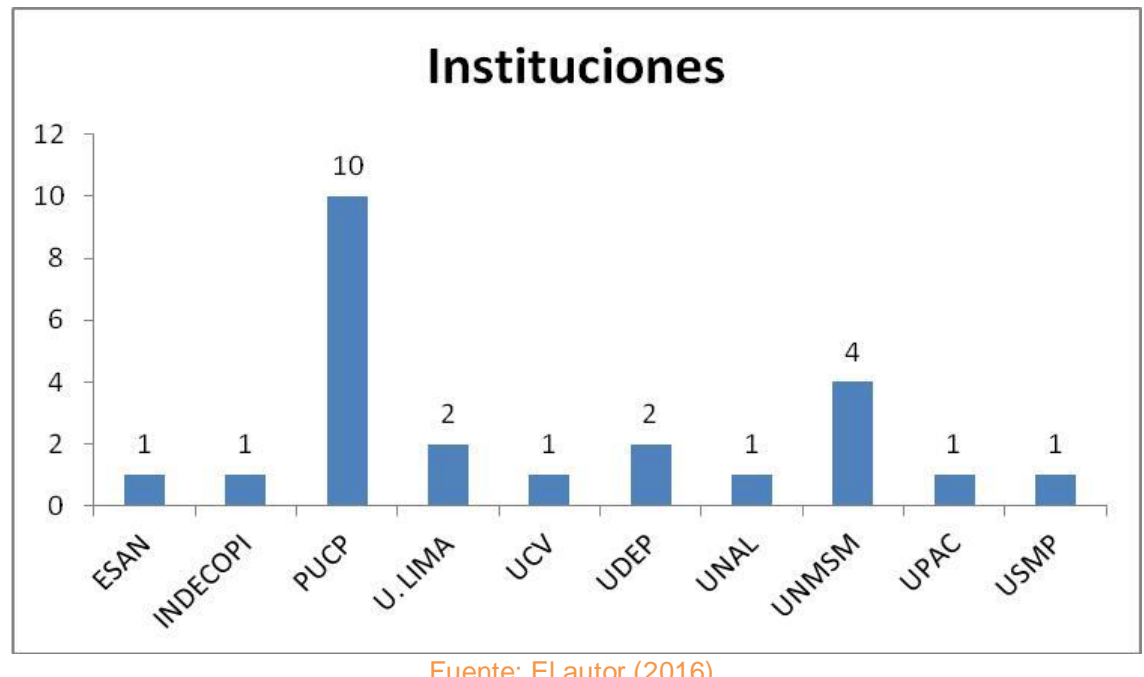

Del total de publicaciones (ver Gráfico 3) 18 corresponden a universidades privadas y 5 a universidades públicas. La única revista independiente está a cargo del Instituto Nacional de Defensa de la Competencia y de la Protección de la Propiedad Intelectual (Indecopi).

\section{Gráfico 3: Naturaleza de la institución}

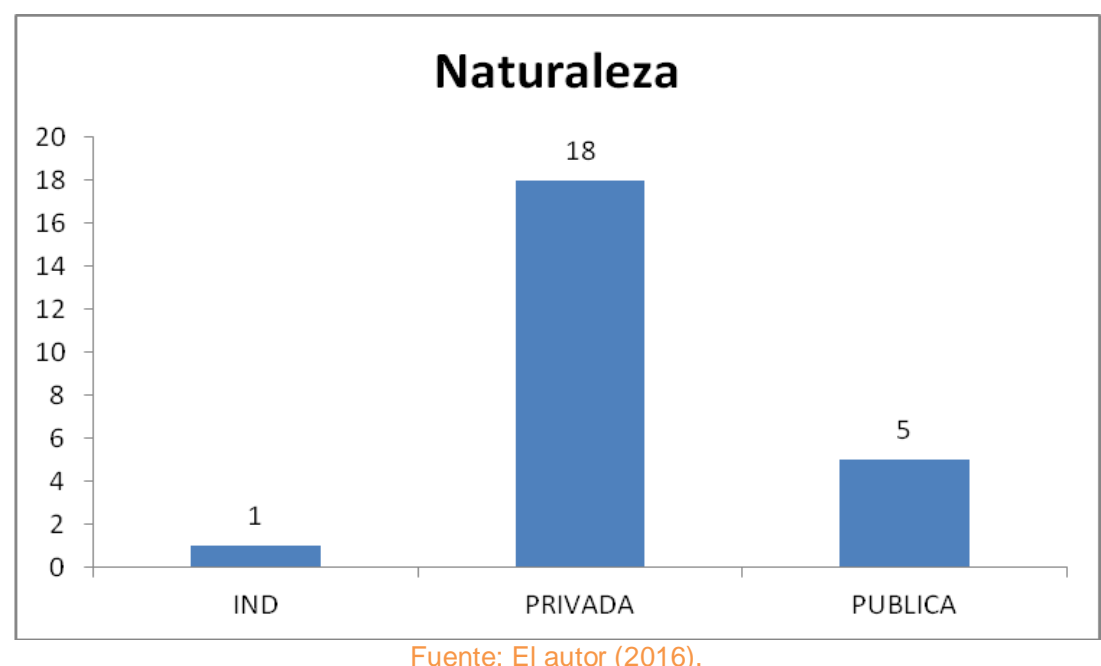

5.1.4 Frecuencia de la publicación: 12 revistas se publican anualmente, mientras que las 12 restantes lo hacen con una periodicidad de 2 números por año. 


\subsection{Campo 2: Producción científica}

En total se registran 2791 artículos publicados entre los años 2005-2014.

5.2.1 Artículos publicados: Como puede observarse en el siguiente gráfico (ver Gráfico 4), la revista que más artículos ha publicado es Investigaciones Sociales (UNMSM) con 333 artículos. Le sigue Derecho (PUCP) con 227 contribuciones, Investigación Psicológica con 179, Liberabit (USMP) con 154, Quipukamayoc con 146. Con 130 figura Contabilidad y Negocio y Cuadernos de Difusión (ESAN), que en el 2009 cambia de nombre por Journal of Economics.

Con algo más de 100 artículos se ubica la Revista de Derecho (UDEP) (110) e Investigación Educativa (UNMSM) con 109. En un grupo más compacto observamos 14 revistas con una productividad que varía entre 80 y 90 artículos durante el período. Finalmente las que menos artículos han publicado son Comunic@acción (UAL) con 52 y Debates de Sociología (PUCP) con 50.

Hay que indicar que el bajo número de artículos de algunas revistas obedece a que solo editan un volúmen por año. También porque hemos observado casos de discontinuidad en algunos números que simplemente no fueron publicados o fueron publicados con retraso, afectando su periodicidad habitual. Es el caso de Boletín de Arqueología, Comunic@ción, Journal of Centrum (PUCP), que comienza a editarse en 2008 y finalmente la Revista de Psicología (UCV).

\section{Gráfico 4: Número de artículos por revista}

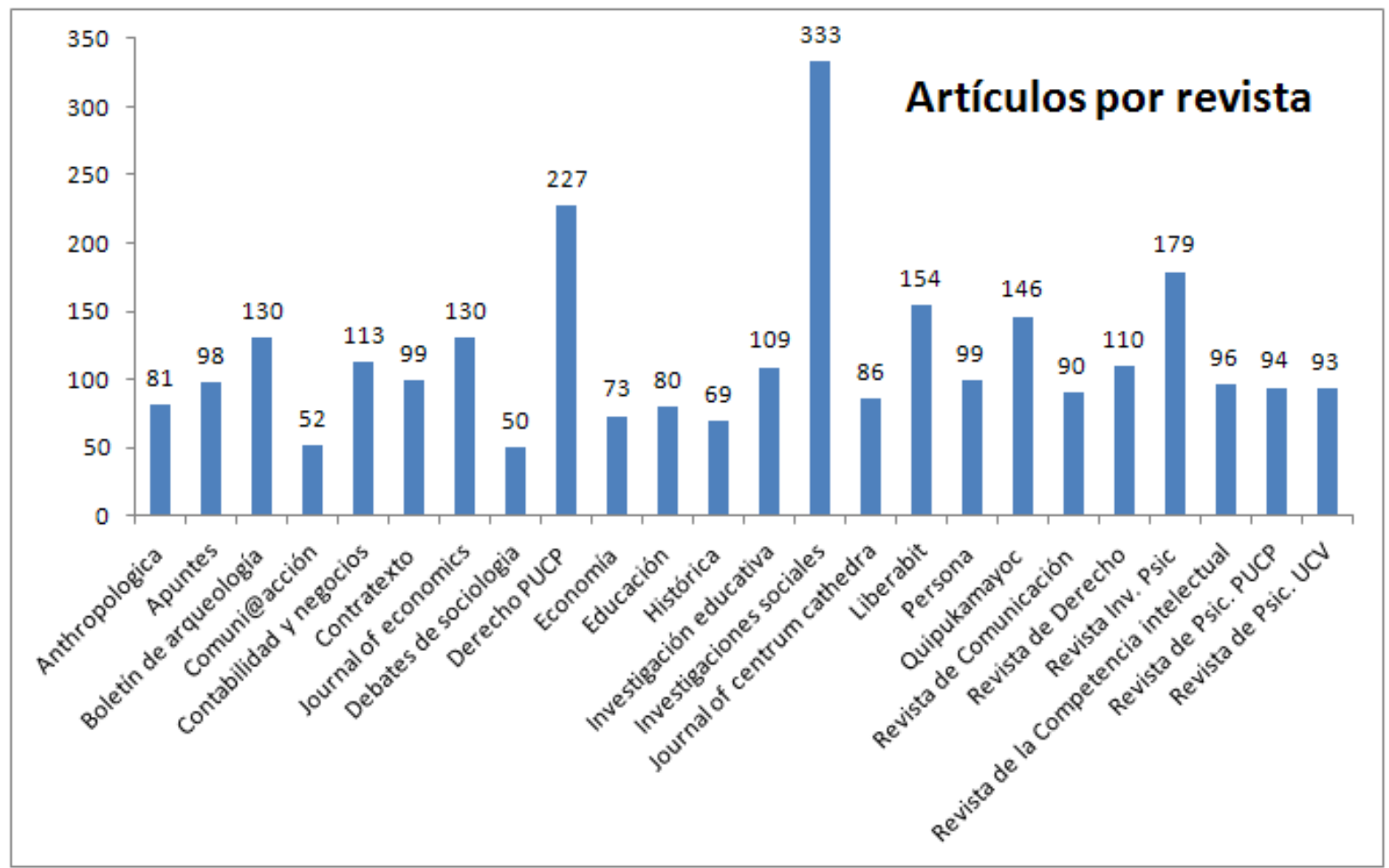

Fuente: El autor (2016).

En el siguiente gráfico (ver Gráfico 5) puede observarse la producción numérica de todas las publicaciones durante el intervalo analizado. 


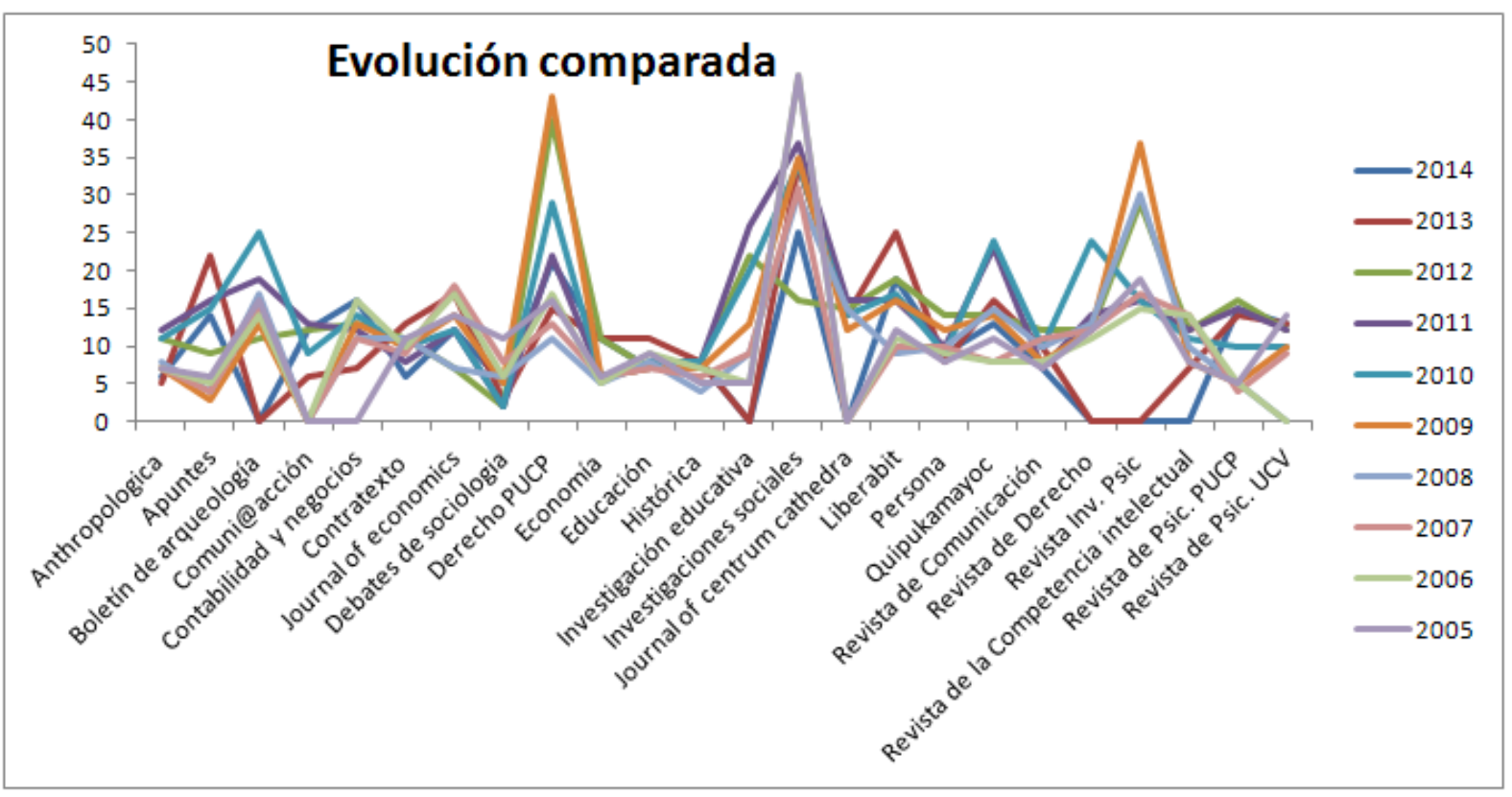

Fuente: El autor (2016).

Para apreciar con mayor detalle las tendencias evolutivas de la producción de artículos los datos correspondientes a cada revista se desglosan anualmente en la siguiente tabla (ver Tabla 3).

Tabla 3: Evolución comparada

\begin{tabular}{|l|c|c|c|c|c|c|c|c|c|c|c|c|c|c|c|}
\hline REVISTA & UNIVINST. 2014 & 2013 & 2012 & 2011 & 2010 & 2009 & 2008 & 2007 & 2006 & 2005 & ARTICULOS \\
\hline Anthropologica & PUCP & 6 & 5 & 11 & 12 & 11 & 7 & 8 & 7 & 7 & 7 & 81 \\
\hline Apuntes & UPAC & 14 & 22 & 9 & 16 & 15 & 3 & 4 & 4 & 5 & 6 & 98 \\
\hline Boletín de arqueología & PUCP & 0 & 0 & 11 & 19 & 25 & 13 & 17 & 15 & 14 & 16 & 130 \\
\hline Comuni@acción & UNAL & 12 & 6 & 12 & 13 & 9 & 0 & 0 & 0 & 0 & 0 & 52 \\
\hline Contabilidad y negocios & PUCP & 16 & 7 & 13 & 12 & 14 & 13 & 11 & 11 & 16 & 0 & 113 \\
\hline Contratexto & U. LIMA & 6 & 13 & 11 & 8 & 10 & 10 & 11 & 9 & 10 & 11 & 99 \\
\hline Journal of economics & ESAN & 12 & 17 & 7 & 12 & 12 & 14 & 7 & 18 & 17 & 14 & 130 \\
\hline Debates de sociologia & PUCP & 5 & 3 & 2 & 2 & 2 & 5 & 6 & 8 & 6 & 11 & 50 \\
\hline Derecho PUCP & PUCP & 21 & 15 & 40 & 22 & 29 & 43 & 11 & 13 & 17 & 16 & 227 \\
\hline Economía & PUCP & 11 & 11 & 11 & 6 & 6 & 6 & 5 & 6 & 5 & 6 & 73 \\
\hline Educación & PUCP & 7 & 11 & 7 & 7 & 8 & 7 & 8 & 7 & 9 & 9 & 80 \\
\hline Histórica & PUCP & 8 & 8 & 8 & 8 & 8 & 7 & 4 & 6 & 7 & 5 & 69 \\
\hline Investigación educativa & UNMSM & 0 & 0 & 22 & 26 & 20 & 13 & 9 & 9 & 5 & 5 & 109 \\
\hline Investigaciones sociales & UNMSM & 25 & 34 & 16 & 37 & 34 & 35 & 30 & 31 & 46 & 46 & 333 \\
\hline Journal of centrum cathedra & PUCP & 0 & 14 & 15 & 16 & 14 & 12 & 15 & 0 & 0 & 0 & 86 \\
\hline Liberabit & USMP & 19 & 25 & 19 & 16 & 17 & 16 & 9 & 10 & 11 & 12 & 154 \\
\hline Persona & U. LIMA & 9 & 8 & 14 & 10 & 9 & 12 & 10 & 10 & 9 & 8 & 99 \\
\hline Quipukamayoc & UNMSM & 13 & 16 & 14 & 23 & 24 & 14 & 15 & 8 & 8 & 11 & 146 \\
\hline Revista de Comunicación & UDEP & 7 & 10 & 12 & 7 & 10 & 8 & 10 & 11 & 8 & 7 & 90 \\
\hline Revista de Derecho & UDEP & 0 & 0 & 12 & 14 & 24 & 12 & 13 & 12 & 11 & 12 & 110 \\
\hline Revista Inv. Psic & UNMSM & 0 & 0 & 29 & 16 & 16 & 37 & 30 & 17 & 15 & 19 & 179 \\
\hline Revista de la Competencia in INDECOPI & 0 & 7 & 12 & 12 & 11 & 8 & 10 & 14 & 14 & 8 & 96 \\
\hline Revista de Psic. PUCP & PUCP & 15 & 14 & 16 & 15 & 10 & 5 & 5 & 4 & 5 & 5 & 94 \\
\hline Revista de Psic. UCV & UCV & 13 & 13 & 12 & 12 & 10 & 10 & 0 & 9 & 0 & 14 & 93 \\
\hline
\end{tabular}


En el presente cuadro podemos advertir oscilaciones significativas en cuanto al número total de artículos publicados por año: Antropolhogica (5-12), Contabilidad y negocios (7-16), Contratexto (6-13), Journal of Economics (7-18), Educación (7-9) y Persona (8-14). Con tendencia creciente en el tiempo vemos a Apuntes (de 6 a 22 artículos), Economia (de 6 a 11), Histórica (5 y 8), Liberabit (11 y 25) y Revista de Psicología (5 y 16). Finalmente, con tendencia a la baja encontramos a: Investigaciones sociales (46 a 25 artículos), Boletín de Arqueología (16 a 11 artículos) y Revista de la Competencia intelectual (14 a 7).

\subsubsection{Autores}

Hay un total de 4018 autores, 2432 nacionales y 1586 extranjeros. Esta cifra representa el 65,58\% de autores nacionales y $34,42 \%$ de autores extranjeros (ver las columnas 2 a 5 de la Tabla III). La revista con más autores nacionales es Investigaciones sociales con 382, seguida de Revista de investigación psicológica con 363. Ambas son editadas por la Universidad de San Marcos (UNMSM). Luego se encuentra Derecho con 177 (UDEP) y Quipukamayoc (UNMSM) con 171. Las que registran menos autores peruanos son Comunicación (UDEP) con 20 e Histórica (PUCP) con 13.

Respecto de autores extranjeros el número más elevado lo posee Liberabit (USMP) con 217, seguido de la Revista de Psicología (PUCP) con 152. Luego Centrum Cathedra con 132 y el Boletín de Arqueología con 120, las tres revistas pertenecen a la PUCP. Las que registran menor número de contribuciones provenientes del extranjero son: Comunic@acción (UL) con 16 artículos, Quipukamayoc (UNMSM) con 11, Debates de Sociología (PUCP) con 13. Si analizamos los datos en términos porcentuales, vemos que la Revista de Comunicación (UDEP) alcanza un 83,8\% de autores extranjeros. Sigue Centrum Cathedra con 82,5\%, Liberabit (USMP) un 73,8\% y Revista de Psicología (PUCP) con un 71,3\%. Las que poseen menos número de autores extranjeros son Quipucamayoc con 7,6\% e Investigaciones sociales con solo un 2,3\%, ambas de la Universidad Mayor de San Marcos. Se detecta además, en ambas publicaciones una alta concentración de autores de la propia universidad que inclusive han publicado varias veces en una misma revista.

\subsubsection{Internacionalidad de las contribuciones}

Otro indicador asociado a la calidad es el número de artículos donde participan simultáneamente autores nacionales y extranjeros (ver las dos últimas columnas de la Tabla III). En esta variable, la revista que mejor refleja esta forma de coautoría es el Boletín de arqueología con 19 artículos, seguido de Psicología con 6, ambas de la Pontificia Unversidad Católica. Algunos casos aislados de esta fórmula de autoría también se registra en Liberabit (USMP) con 5, Apuntes (UDEP) con 3, al igual que Economía. 10 revistas no registran contribuciones donde participan investigadores nacionales juntamente con extranjeros.

Según su procedencia, España representa el país con mayor número de artículos publicados y tiene presencia en revistas de todos los campos: Revista de Comunicación 50\%; Revista de Derecho 35,7; Contabilidad y negocios 26,53\%; Histórica 22,2\%; Contratexto 16,36\%; CD Journal of economics 12,4\%; Educación 11,5\%. Sigue Estados Unidos: Journal of Centrum 24,3\%; Boletín de Arqueología 22,5\%; Histórica 22,2\% o Economía 13,6\%. Con menor tasa de contribuciones se encuentran artículos procedentes de otros países de América Latina como México, Brasil, Argentina o Chile.

Atendiendo a la procedencia de las contribuciones, podemos detectar un total de 56 contribuciones científicas presentadas conjuntamente por investigadores extranjeros y peruanos (ver última columna de la Tabla 3). La revista Boletín de Arqueología posee 19 artículos elaborados por autores de Perú-Estados Unidos (9), 4 PerúAlemanía y 2 Perú-Japón. El resto corresponden a coautorías con otros países como España, Argentina o Chile, por citar los más frecuentes. Journal of Centrum y Liberabit incluyen un artículo publicado entre PerúIndia. Revista de Psicología y Liberabit reportan uno entre Perú-Bélgica. 
Tabla 4: Producción por autores y países

\begin{tabular}{|c|c|c|c|c|c|c|c|}
\hline Revista & A N & $\%$ & $A E X$ & $\%$ & Países $\%$ del total & $A N+A E X$ & Paises \\
\hline Anthropológica & 27 & $35,21 \%$ & 52 & $64,78 \%$ & $\begin{array}{l}\text { AR: } 16,46 \% \\
\text { ES: } 11,39 \% \\
\text { US: } 10,13 \%\end{array}$ & 1 & $\mathrm{PE}+\mathrm{FR}(1)$ \\
\hline Apuntes & 32 & $49,23 \%$ & 33 & $50,77 \%$ & $\begin{array}{l}\text { US: } 18,07 \% \\
\text { AR: } 12,05 \% \\
\text { BR: } 7,23 \%\end{array}$ & 3 & $\mathrm{PE}+\mathrm{US}(3)$ \\
\hline Boletín de arqueología & 69 & $36,5 \%$ & 120 & $63,4 \%$ & $\begin{array}{l}\text { EU: } 22,5 \% \\
\text { DE: } 12,74 \% \\
\text { JP: } 8,09 \%\end{array}$ & 19 & \begin{tabular}{|l|}
$\mathrm{PE}+\mathrm{US}(9)$ \\
$\mathrm{PE}+\mathrm{DE}(4)$ \\
$\mathrm{PE}+\mathrm{JP}(2)$ \\
\end{tabular} \\
\hline Comunic@acción & 64 & $80,0 \%$ & 16 & $20,0 \%$ & - & 0 & - \\
\hline Contabilidad y negocios & 72 & $42,6 \%$ & 97 & $57,4 \%$ & $\begin{array}{l}\text { ES: } 26,63 \% \\
\text { CL: } 8,88 \\
\text { BE: } 8,88 \%\end{array}$ & 4 & $\begin{array}{c}\mathrm{UR}+\mathrm{AR}+\mathrm{P} \\
(1) \\
\mathrm{BR}+\mathrm{PE}(1) \\
\mathrm{ES}+\mathrm{PE}+\mathrm{AR} \\
\text { (1) }\end{array}$ \\
\hline Contratexto & 47 & $42,7 \%$ & 63 & $57,3 \%$ & $\begin{array}{l}\text { ES: } 16,36 \% \\
\text { MX: } 12,73 \% \\
\text { AR: } 8,18 \%\end{array}$ & 3 & $\begin{array}{l}\mathrm{PE}+\mathrm{AR}(2) \\
\mathrm{ES}+\mathrm{CL}(1) \\
\end{array}$ \\
\hline Journal of economics & 57 & $29,5 \%$ & 101 & $70,4 \%$ & $\begin{array}{l}\text { ES: } 12,4 \% \\
\text { CO: } 10,8 \% \\
\text { MX: } 7,25 \%\end{array}$ & 2 & $\begin{array}{l}E S+P E(1) \\
C L+E S(1)\end{array}$ \\
\hline Debates de sociología & 40 & $75,4 \%$ & 13 & $24,6 \%$ & $\begin{array}{l}\text { EU: } 11,36 \% \\
\text { AR: } 5,6 \% \\
\text { MX: } 3,7 \% \\
\end{array}$ & 0 & - \\
\hline Derecho PUCP & 177 & $64,5 \%$ & 67 & $24,4 \%$ & $\begin{array}{l}\text { ES: } 10,60 \% \\
\text { AR: } 4,51 \% \\
\text { IT: } 2,87 \% \\
\end{array}$ & 3 & $\begin{array}{l}\mathrm{PE}+\mathrm{CH}(2) \\
\mathrm{PE}+\mathrm{BR}(1)\end{array}$ \\
\hline Economía & 61 & $69,3 \%$ & 27 & $30,6 \%$ & $\begin{array}{l}\text { US: } 13,6 \% \\
\text { AR: } 5,6 \% \\
\text { ES: } 4,5 \%\end{array}$ & 3 & $\begin{array}{l}\mathrm{PE}+\mathrm{ES}(2) \\
\mathrm{ES}+\mathrm{PE}(1)\end{array}$ \\
\hline Educación & 71 & $68,2 \%$ & 33 & $31,8 \%$ & $\begin{array}{l}\text { ES: } 11,54 \% \\
\text { BR: } 6,7 \% \\
\text { AR: } 3,8 \% \\
\end{array}$ & 2 & $\mathrm{PE}+\mathrm{BR}(2)$ \\
\hline Histórica & 13 & $18 \%$ & 49 & $72 \%$ & $\begin{array}{l}\text { EU: } 22,2 \% \\
\text { ES: } 16,77 \% \\
\text { AR: } 13,89 \%\end{array}$ & 0 & - \\
\hline Investigación educativa & 135 & $85,9 \%$ & 23 & $14,1 \%$ & $\begin{array}{l}\text { ES: } 7,01 \% \\
\text { VE: } 5,10 \% \\
\text { BR: } 1,91 \%\end{array}$ & 0 & - \\
\hline Investigaciones sociales & 382 & $98,7 \%$ & 19 & $2,3 \%$ & $\begin{array}{l}\text { ES: } 1,34 \% \\
\text { MX: } 0,81 \% \\
\text { AR: } 0,54 \% \\
\end{array}$ & 0 & - \\
\hline $\begin{array}{l}\text { Journal of Centrum } \\
\text { cathedra }\end{array}$ & 28 & $17,5 \%$ & 132 & $82,5 \%$ & $\begin{array}{l}\text { EU: } 24,3 \% \\
\text { ES: } 8,75 \% \\
\text { UK: } 6,88 \% \\
\end{array}$ & 3 & \begin{tabular}{|l|} 
PE-EU (1) \\
PE-ES. (1) \\
PE-IN. (1)
\end{tabular} \\
\hline Liberabit & 77 & $26,1 \%$ & 217 & $73,8 \%$ & $\begin{array}{l}\text { AR: } 22,11 \% \\
\text { MX: } 17,01 \% \\
\text { BR: } 13,27 \%\end{array}$ & 5 & \begin{tabular}{|l|} 
PE-EU (1) \\
PE-BE (1) \\
BR-PE (1) \\
\end{tabular} \\
\hline Persona & 65 & $50,0 \%$ & 65 & $50,0 \%$ & $\begin{array}{l}\text { BR: } 10,77 \% \\
\text { ES: } 9,23 \% \\
\text { MX: } 6,92 \%\end{array}$ & 2 & $\begin{array}{l}\text { PE-EU (1) } \\
\text { PE-CL (1) }\end{array}$ \\
\hline Quipukamayoc & 171 & $92,4 \%$ & 14 & $7,66 \%$ & $\begin{array}{l}\text { MX: } 6,49 \% \\
\text { AR: } 0,54 \% \\
\text { GT: } 0.54 \%\end{array}$ & 0 & - \\
\hline Revista de Comunicación & 20 & $16,1 \%$ & 104 & $83,8 \%$ & $\begin{array}{l}\text { ES: } 50 \% \\
\text { CL: } 8,06 \% \\
\text { BRI: } 6,45 \% \\
\end{array}$ & 0 & - \\
\hline Revista de Derecho & 174 & $71,3 \%$ & 51 & $28,6 \%$ & $\begin{array}{l}\text { ES: } 35,7 \% \\
\text { AR: } 3,67 \% \\
\text { IT: } 1,83 \%\end{array}$ & 3 & $\begin{array}{l}\text { PE-CL (2) } \\
\text { PE-BR (1) }\end{array}$ \\
\hline $\begin{array}{l}\text { Revista Investigación } \\
\text { Psicológica }\end{array}$ & 363 & $84,6 \%$ & 66 & $15,4 \%$ & $\begin{array}{l}\text { MX: } 3,96 \% \\
\text { CL: } 3,03 \% \\
\text { BR: } 2,33 \% \\
\end{array}$ & 0 & - \\
\hline $\begin{array}{l}\text { Revista de la Competencia } \\
\text { Intelectual }\end{array}$ & 100 & $83,3 \%$ & 20 & $16,7 \%$ & $\begin{array}{l}\text { AR: } 10 \% \\
\text { CH: } 1,67 \% \\
\text { EU: } 1,67 \% \\
\end{array}$ & 0 & - \\
\hline Revista de Psicología PUCP & 61 & $28,7 \%$ & 152 & $71,3 \%$ & $\begin{array}{l}\text { AR: } 18,31 \% \\
\text { BR: } 10,77 \% \\
\text { ES: } 8,45 \%\end{array}$ & 3 & \begin{tabular}{|l|} 
PE-BE (2) \\
PE-EU (1) \\
PE-BL (1)
\end{tabular} \\
\hline Revista de Psicología UCV & 126 & $75,4 \%$ & 52 & $31,1 \%$ & $\begin{array}{l}\text { BR: } 11,9 \% \\
\text { AR: } 7,8 \% \\
\text { ES: } 4,79 \%\end{array}$ & 0 & PE-BE (1) \\
\hline TOTAL REVISTAS: 24 & 242 & & 1586 & & & 56 & \\
\hline
\end{tabular}


Accediento al portal Redalyc, hemos podido obtener información acerca de la procedencia de las contribuciones de 5 revistas por centros. Este indicador nos permite apreciar los nexos de las revistas con otras universidades peruanas y del exterior. En la siguiente tabla (ver Tabla 4), visualizamos, en primer lugar, un predominio muy marcado de autores de los propios centros. La tendencia endogénica más clara es en Contabilidad y negocios (PUCP) con un 41,4\% de artículos elaborados por investigadores de la propia universidad. Sigue Persona (UL) con 32,5\%; luego Journal of economics con 22,5\%; (Centrum PUCP), Revista de Psicología (PUCP) con 21,1\% y finalmente Liberabit (USMP) con 19,4\%. Sin embargo, oberservando con detalle la procedencia de las contribuciones, comprobamos que Contabilidad y negocios (PUCP) posee vínculos estrechos con universidades españolas, dando acogida a un número importante de artículos: Universidad de Valencia 7,1\%, Universidad de Vigo y la Universidad de Zaragoza, ambas con 4,0\%. Por su paarte, Journal of economics se mueve preferentemente en un espacio más latinoamericano: EAFIT (Colombia) 6\%; México ITAM y ITESM con 2,6\%. Reporta también un $6 \%$ de artículos de la Universidad Sfax de Túnez. Liberabit (USMP), por su parte, acoge contribuciones procedentes de diferentes universidades de América Latina como UNAM (México) 7,9; Pontificia Universidad Católica (Perú) 5\% y la Universidad de Concepción (Chile) 4,3\%. La Persona (UL) concentra fuertemente sus índices de autoría en investigadores nacionales. En cuanto a los extranjeros, regista solo un $3 \%$ de artículos de la UNAM (México). Finalmente, la Revista de Psicología (PUCP) incluye artículos de Argentina: Consejo Nacional de Investigaciones Científicas y Técnicas, 6,8\% y la Universidad de Buenos Aires: 4,3\%. También de la Universidad de Brasilia, 4,3\% y México 2,0\%, concretamente de la Universidad de Sonora.

Tabla 5: Instituciones contribuyentes

\begin{tabular}{|l|l|}
\hline \multicolumn{1}{|c|}{ Revista } & \multicolumn{1}{|c|}{ Instituciones contribuyentes } \\
\hline Contabilidad y negocios & PUCP: 41,4\%; U. Valencia: 7,1\%; U. Vigo: 4,\%; U. Zaragoza: 4,0\% \\
\hline CD J.of economics & ESAN: 22,5\%; EAFIT: 6,0\%; U. Sfax: 4,0\%; ITAM: 2,6\%; ITENSM: 2,6\% \\
\hline Liberabit & USMP: 19,4\%; UNAM: 7,9\%; PUCP: 5,0\%; UC: 4,3\%; otras: 59,0\% \\
\hline Persona & UL: 32,5\%; UNMSM: 5,2\%; C. Per. Aud. y Leng. 3\%; UNAM: 3\% \\
\hline Revista de Psic. PUCP & PUCP: 21,1\%; CNI: 6,8\%; UBA: 4,3\%; U. Brasilia: 4,3\%; U. Sonora 2,0\% \\
\hline
\end{tabular}

\subsection{Campo 3: Indicadores de calidad internacional}

En este apartado analizamos las citaciones a los artículos de las revistas producidas durante los años 20042014 (ver Tabla V). Para ello empleamos como fuentes: Citaciones en Google Académico (GAC), Clase y SciELO. Se incluye el FI SciELO al 2013 y los Criterios Latindex cumplidos.

La revista con mayor número de citaciones en Google Académic es Liberabit (USMP) con 1130 citaciones, seguida de Debates en Sociología (PUCP) con 985. En tercer lugar encontramos al Boletín de Arqueología, (PUCP) con 931. En un segundo grupo, está Histórica (PUCP) con 592; Persona (UL) con 577; Journal of Centrum: 427 y Antropológica con 346.

Números inferiores, pero que mantienen una cierta proporcionalidad con Google Académico, son los que se muestran en las citaciones que almacena CLASE. Por ejemplo, aquí la más citada, y con gran holgura, es Histórica que reporta 448 citas, le sigue Antropológica con 255. Más abajo vemos a Derecho con 140, las tres de la Universidad Católica. Luego encontramos a Apuntes (UPAC) con 177, Boletín de Arqueología con 136 y muy cerca, con 134 Debates de Sociología y Derecho con 111, estas tres últimas de la PUCP. Liberabit que en GAC tiene 1130 aquí solo reporta 99 citas. Debemos precisar que las citas de GAC se recogen de todo el tiempo de existencia de la revista y no únicamente del período seleccionado para este estudio.

Los 10 registros de citas ofrecidos por CLASE son muy inferiores respecto de GAC (ver tercera columna de la Tabla V). Podemos visualizar que la revista con mayor número de citaciones es Histórica con 448, seguida de Anthropológica con 255. Seguidamente se ubica Economia y Debates de Sociología con 139 y 136 citas, respectivamente. Continúa Derecho con 111. Estas 5 revistas pertenecen a la Universidad Católica (PUCP). Con 99 citas tenemos a Liberabit (USMP), Contabilidad y Negocios con 81 (PUCP), Apuntes (UPAC) con 28. Con 21 se ubica Investigación Educativa (UNMSM) la única revista editada por una universidad pública. Cerrando la lista tenemos a la Revista de Psicología de la Universidad Católica con 10 citaciones. Debemos indicar que a diferencia de los datos de GAC que nos fueron datos totales de la revista, en el buscador de 
CLASE fue posible hacer una búsqueda acotada a solo las citas entre los años 2005-2014. Esto puede explicar en parte las diferencias observadas entre ambas fuentes.

Por su parte, el buscador SciELO nos muestra métricas de citas de 4 revistas que indican valores muy inferiores respecto de las ofrecidas por GAC y CLASE. En SciELO sólo aparecen 6 revistas. La Revista de Psicología obtiene el primer lugar del ranquin con 450 citaciones, es seguida por Liberabit que obtiene 110, luego se posiciona Anthropológica con 83. En antepenúltima posición tenemos a Journal of Economics con 15 y cerrando la lista Comunic@ción (UAL) y Apuntes (UPAC), que no registan citas.

SciELO, además genera una estimación de factor de impacto (IF), basado en el volumen y procedencia de las citaciones. La mejor posicionada de las revistas nacionales es Liberabit con 0,0676, seguida de Antropológica con 0,0323. En tercer lugar se ubica Journal of economics que llega a 0,055 y finalmente la Revista de Psicología obtiene el 0,023. Apuntes y Comunic@cción, no registra información de IF, por no haber recibido cita alguna en los últimos 3 años.

En cuanto a los criterios Latindex cumplidos (CLI) -ver última columna de la Tabla 6-, solo Apuntes cumple con el total de 36. Con 33 igualan 5 revistas: Comunic@acción, Educación, Liberabit, Revista de Comunicación y Revista de Psicología (PUCP). Anthropológica, Persona y Revista de Psicología (UCV) tienen 32. Le siguen con 31, Debates en sociología, Economía, Investigación educativa y Quipukamayoc. 3 obtienen 30: Journal of economics, Contratexto y la Revista de Investigación en psicología. En un segundo grupo observamos al Boletín de arqueología, Investigaciones sociales y Journal of Centrum cathedra, con 29. Las que menos criterios cumplen son Histórica con 27 y la Revista de la Competencia intelectual, con tan solo 25. Esperábamos hallar una correspondencia directa entre citas, $\mathrm{FI}$ y el número de Criterios Cumplidos de Latindex que no pudimos constatar.

Tabla 6: Citaciones en Google Académico (GAC), Clase, Scielo, factor de impacto Scielo (IF Scielo 2013) y Criterios Latindex cumplidos (CLI).

\begin{tabular}{|c|c|c|c|c|c|}
\hline Revista & GAC & CLASE & SCIELO & $\begin{array}{c}F \\
\text { SCIELO } \\
2013\end{array}$ & CLI \\
\hline Anthropológica & 346 & 255 & 83 & 0,0323 & 32 \\
\hline Apuntes & 24 & 28 & 0 & 0 & 36 \\
\hline Boletín de arqueología & 931 & 136 & - & - & 29 \\
\hline Comunic@acción & - & - & 0 & 0 & 33 \\
\hline Contabilidad y negocios & 122 & 81 & - & - & 32 \\
\hline Contratexto & 272 & - & - & - & 30 \\
\hline Journal of economics & 145 & - & 15 & 0,055 & 30 \\
\hline Debates en sociología & 985 & 134 & - & - & 31 \\
\hline Derecho PUCPiii & 96 & 111 & - & - & 29 \\
\hline Economía & 75 & 139 & - & - & 31 \\
\hline Educación & 4 & - & - & - & 33 \\
\hline Histórica & 592 & 448 & - & - & 27 \\
\hline Investigación educative & 115 & 21 & - & - & 31 \\
\hline Investigaciones sociales & 112 & - & - & - & 29 \\
\hline Journal of Centrum cathedra & 427 & - & - & - & 29 \\
\hline Liberabit & 1130 & 99 & 110 & 0,07 & 33 \\
\hline Persona & 577 & - & - & - & 32 \\
\hline Quipukamayoc & 10 & - & - & - & 31 \\
\hline R. de Comunicación & - & - & - & - & 33 \\
\hline R. de Derecho & 99 & - & - & - & 31 \\
\hline R. Investigacion en psicología & - & - & - & - & 30 \\
\hline R. de la Competencia Intelectual & 51 & - & - & - & 25 \\
\hline R. de Psicología PUCPiv & 167 & 10 & 450 & 0,023 & 33 \\
\hline R. de Psicología UCV & 3 & - & - & - & 32 \\
\hline
\end{tabular}

En la siguiente tabla (Tabla 7) vemos las visitas al portal, el número de artículos con mayor descarga. También el número y porcentaje por países de donde provienen estas descargas. Esta información, solo parcial para algunas revistas, ha sido facilitada gentilmente por los administradores de los portales web de las revistas de la Pontificia Universidad Católica, Universidad de Lima y Universidad de Piura ${ }^{\text {vi }}$, ya que no está disponible en abierto. Los datos Journal of economics y Journal of Centrum Cathedra, se obtienen a través de los portales LogEC o Ideas debido a que tampoco son accesibles a través de Internet. 
Vemos primeramente las visitas a los portales de las revistas. La revista más visitada es Revista de Psicología con 197,918 accesos, sieguida de Derecho con 115,334. En tercer lugar se ubica Educación con 100,187 y cuarta Contabilidad y negocio con 80,918. Las restantes no alcanzan las diez mil visitas.

En cuanto al volumen total de artículos descargados. El primer lugar lo ocupa Derecho con 191,627, seguido de Revista de Psicología con 173,904. En tercer lugar, prácticamente igualados, tenemos a Histórica con 132,743 y Educación con 132,116 descargas totales de artículos. Más abajo vemos a Contabilidad y negocios con 57,329, todas de la Universidad Católica.

El mayor número de descargas de un artículo lo tiene la Revista de Comunicación (UDEP) con 2498, le sigue Contratexto (UL) con 2240, ambas del ámbito de las Ciencias de la Comunicación. En un tercer bloque vemos a Liberabit, 2050. Más lejos está Contabilidad y negocios con 932 y Revista de Derecho con 459 ambas de la Universidad Católica. Las siguientes tienen descargas inferiores.

En cuanto al país de origen de las descargas tenemos que la revista con mayor número de artículos descargados del propio país es Derecho (UDEP) con un 88,44\%, seguido de Contratexto con 59,6\%. Con $48,14 \%$ está la Revista de Comunicación. Las que registran un menor número de descargas nacionales Contabilidad y Negocios 11,8\%, seguida muy cerca por Revista de Psicología con 11,6\% y Liberabit con solo $8,2 \%$, lo que indica que sus lectores son principalmente extranjeros.

Finalmente examinemos las revistas con más descargas de artículos desde el extranjero. Nuevamente la Revista de Psicología de la PUCP lidera el cuadro con el 43,1\% de descargas registradas principalmente desde México. También con un 32,3\% desde ese país tenemos a Contabilidad y negocios y Liberabit con 29\%. Estos datos reflejan una importante presencia de estas revistas en universidades de dicho país. De hecho, si relacionamos la información con los datos del cuadro anterior, vemos que existe una correlación importante, pues un gran número de contribuciones a revistas peruanas provienen de investigadores de universidades de México. También hay descargas importantes desde Argentina. En Contratexto un 7,13\% y en la Revista de Derecho: 2,31\%. Las restantes provienen de Chile y Estados Unidos: 10,2\% de la revista Contabilidad y Negocios, Persona con 9,8\% y Liberabit 9,7\%, respectivamente. Un dato relevante y que confirma una vez más la diferencia en los datos reportados, es que el registro de descargas es distinto según la PUCP $(57,329)$ y Redalyc (33, 019).

Tabla 7: Descargas de artículos por países: Descargas recibidas hasta el mes de noviembre de 2014 de artículos publicados dentro del período 2005-2014.

\begin{tabular}{|c|c|c|c|c|c|}
\hline Revista & $\begin{array}{c}\text { Visitas al } \\
\text { portal }\end{array}$ & $\begin{array}{l}\text { Total } \\
\text { desc. }\end{array}$ & № de desc. & País & $\%$ \\
\hline \multirow{3}{*}{ Contabilidad y negocios } & \multirow{3}{*}{80,918} & \multirow{3}{*}{$\begin{array}{l}57,329 v i i \\
33,019 v i i i\end{array}$} & 932 & México & $32,3 \%$ \\
\hline & & & 339 & Perú & $11,8 \%$ \\
\hline & & & 295 & EU & $10,2 \%$ \\
\hline \multirow{3}{*}{ Contratexto } & \multirow{3}{*}{6080} & \multirow{3}{*}{3757} & 2240 & Perú & $59,6 \%$ \\
\hline & & & 268 & Argentina & $7,13 \%$ \\
\hline & & & 267 & México & $7,10 \%$ \\
\hline \multirow{3}{*}{ Journal of economics } & \multirow{3}{*}{-} & \multirow{3}{*}{1202} & - & - & - \\
\hline & & & - & - & - \\
\hline & & & - & - & - \\
\hline \multirow{3}{*}{ Derecho PUCP } & \multirow{3}{*}{115,334} & \multirow{3}{*}{191,627} & - & - & - \\
\hline & & & - & - & - \\
\hline & & & - & - & - \\
\hline \multirow{3}{*}{ Economía } & \multirow{3}{*}{-} & \multirow{3}{*}{4193} & - & - & - \\
\hline & & & - & - & - \\
\hline & & & - & - & - \\
\hline \multirow{3}{*}{ Educación } & \multirow{3}{*}{100,187} & \multirow{3}{*}{132,116} & - & - & - \\
\hline & & & - & - & - \\
\hline & & & - & - & - \\
\hline
\end{tabular}


Tabla 7: Descargas de artículos por países: Descargas recibidas hasta el mes de noviembre de 2014 de artículos publicados dentro del período 2005-2014 (Continuación).

\begin{tabular}{|c|c|c|c|c|c|}
\hline Revista & $\begin{array}{c}\text { Visitas al } \\
\text { portal }\end{array}$ & $\begin{array}{l}\text { Total } \\
\text { desc. }\end{array}$ & № de desc. & País & $\%$ \\
\hline \multirow{3}{*}{ Histórica } & \multirow{3}{*}{69,357} & \multirow{3}{*}{132,743} & - & - & - \\
\hline & & & - & - & - \\
\hline & & & - & - & - \\
\hline Journal Centrum cathedra & - & 1202 & - & - & - \\
\hline \multirow{3}{*}{ Liberabit } & \multirow{3}{*}{-} & & 2050 & México & $29 \%$ \\
\hline & & 125141ix & 686 & EU & $9,7 \%$ \\
\hline & & & 580 & Perú & $8,2 \%$ \\
\hline \multirow{3}{*}{ Persona } & \multirow{3}{*}{7879} & & 682 & México & $25,7 \%$ \\
\hline & & 6329 & 315 & España & $11,9 \%$ \\
\hline & & & 261 & EU & $9,8 \%$ \\
\hline \multirow{3}{*}{ Revista Comunicación } & \multirow{3}{*}{7475} & & 2498 & Perú & $48,14 \%$ \\
\hline & & 7445 & 1054 & España & $20,33 \%$ \\
\hline & & & 379 & México & 7,32 \\
\hline \multirow{3}{*}{ Revista de Derecho } & \multirow{3}{*}{1258} & \multirow{3}{*}{248} & 459 & Perú & $88,44 \%$ \\
\hline & & & 28 & Chile & 5,39 \\
\hline & & & 12 & Argentina & $2,31 \%$ \\
\hline \multirow{3}{*}{ Revista de Psic. PUCP } & \multirow{3}{*}{197,918} & & 286 & México & $43,1 \%$ \\
\hline & & 173,904 & 77 & Perú & $11,6 \%$ \\
\hline & & & 61 & España & $9,2 \%$ \\
\hline
\end{tabular}

Fuente: El autor (2016).

Indexación en otros buscadores

Por último, veamos la visibilidad de las revistas en otros buscadores (ver Tabla 8). 13 revistas son visibles en Dialnet, 6 en SciELO y 6 en Redalyc. Todas las revistas de la Pontificia Universidad Católica se encuentran reunidas en el Portal de Revistas PUCP, mientras que las de la Universidad de San Marcos son accesibles mediante el Sistema Digital de Bibliotecas.

Tabla 8: Indexación en otros buscadores

\begin{tabular}{|c|c|c|c|}
\hline Revistas & DIALNET & SCIELO & REDALYC \\
\hline Anthropológica & $\mathrm{X}$ & $\mathrm{X}$ & $x$ \\
\hline Apuntes & $\mathrm{X}$ & $\mathrm{X}$ & \\
\hline Boletín de arqueología & $x$ & & \\
\hline Comunic@acción & $x$ & $\bar{x}$ & \\
\hline Contabilidad y negocios & $\mathrm{X}$ & & $\mathrm{X}$ \\
\hline Contratexto & $\mathrm{X}$ & & \\
\hline$C D$ J.of economics & & $\mathrm{X}$ & \\
\hline Derecho PUCP & $\mathrm{X}$ & & \\
\hline Economía & $\mathrm{X}$ & & \\
\hline Educación & $\mathrm{X}$ & & \\
\hline Histórica & $x$ & & \\
\hline J. Centrum cathedra & & & $X$ \\
\hline Liberabit & $\mathrm{X}$ & $\mathrm{X}$ & $\mathrm{X}$ \\
\hline Persona & & & $\mathrm{X}$ \\
\hline R. Derecho & $\mathrm{X}$ & & \\
\hline R. Inv. psicología & $\mathrm{X}$ & & \\
\hline Revista de Psic. PUCP & & $\mathrm{X}$ & $\mathrm{X}$ \\
\hline & 13 & 6 & 6 \\
\hline
\end{tabular}

Fuente: El autor (2016).

Si efectuamos una revisión comparativa de citaciones por áreas temáticas (ver Tabla 9), las revistas del ámbito de Ade/Economía/Contabilidad son las que más citas reciben: 6608 en GAC y 482 en CLASE, son también las que más producen con un total de 646 artículos publicados en 6 revistas. Las 2 revistas de Educación alcanzan las 5604 citas en GAC pero solamente 21 en CLASE. En tercera posición se encuentran las de Ciencias Sociales (general) que con solo 2 revistas y 383 artículos alcanzan las 5235 citas en GAC y 134 en CLASE. 
Esto indica que con una producción bastante más baja de comunicaciones consiguen un significativo número de citas. Sigue Derecho, 3 revistas, y la tercera en volumen de artículos del país (433). Obtiene 962 citas en GAC, pero ninguna en CLASE. Las más relegadas son las del área de Comunicación y Psicología con 235 citas y 79 citas respectivamente en GAC. Las de Psicología tienen además 10 citas en CLASE. Véase que el número de citas GAC se obtiene con una producción de 619 artículos, mientras que las de Comunicaciones solo han generado 235, menos de la mitad.

Tabla 9: Comparativa de citas recibidas, revistas y artículos

\begin{tabular}{|l|l|c|c|c|c|}
\hline & & Citas GA & Citas Clase & NoR & Artículos \\
\hline $\mathbf{1}$ & ADE/EC/CONT & $\mathbf{6 6 0 8}$ & $\mathbf{4 8 2}$ & 6 & 646 \\
\hline $\mathbf{2}$ & ARQ/ANTR/HIST. & $\mathbf{5 1 4}$ & $\mathbf{3 9 1}$ & 3 & 280 \\
\hline $\mathbf{3}$ & CIENCIAS SOCIALES & $\mathbf{5 2 3 5}$ & $\mathbf{1 3 4}$ & $\mathbf{2}$ & 383 \\
\hline $\mathbf{4}$ & COMUNICACIÓN & $\mathbf{2 3 5}$ & $\mathbf{0}$ & 3 & 241 \\
\hline $\mathbf{5}$ & DERECHO & $\mathbf{9 6 2}$ & $\mathbf{0}$ & 3 & 433 \\
\hline $\mathbf{6}$ & EDUCACIÓN & $\mathbf{5 6 0 4}$ & $\mathbf{2 1}$ & 2 & 189 \\
\hline $\mathbf{7}$ & PSICOLOGIA & $\mathbf{7 9}$ & $\mathbf{1 0}$ & 5 & 619 \\
\hline
\end{tabular}

En el siguiente gráfico (Gráfico 6) puede verse con mayor nitidez la productividad general de revistas en cada ámbito de conocimiento. La supremacía de ADE en citaciones es muy clara, pero las consigue con un mayor número de revistas y artículos. También, como pudimos ver en la Tabla III, se trata de publicaciones con una alta tasa de autores extranjeros, lo cual puede generar una alta tasa de consultas de sus artículos (visitas, descargas y citas) en centros extranjeros. Sin embargo, ante un número similar de artículos (619) y solo una revista menos (5), el área de Psicología obtiene resultados mucho más bajos: solo 79 citas de GAC y 10 de CLASE. Las de Educación son de hecho las más productivas, tomando como referencia estos indicadores. Ambas, con solo 2 revistas y 189 artículos, tienen más de 5 mil citas de GAC. En CLASE las revistas de Ciencias Sociales llegan solo a 134.

Un dato interesante, es observar que las revistas de Antropología, Arqueología e Historia, son quienes registran un alto número y a la vez bastante cercano de citas en GAC como en CLASE. Han generado 280 papers en 3 revistas. Como puede comprobarse por la altura de la primera barra (cifra de citas GA) comparado con el resto de las áreas e indicadores. En las demás áreas, podemos ver que el número de citas CLASE está más próximo al número de artículos publicados por cada revista (barras segunda y tercera de cada campo).

Grafico 6: Correlación de artículos por ámbitos y citaciones

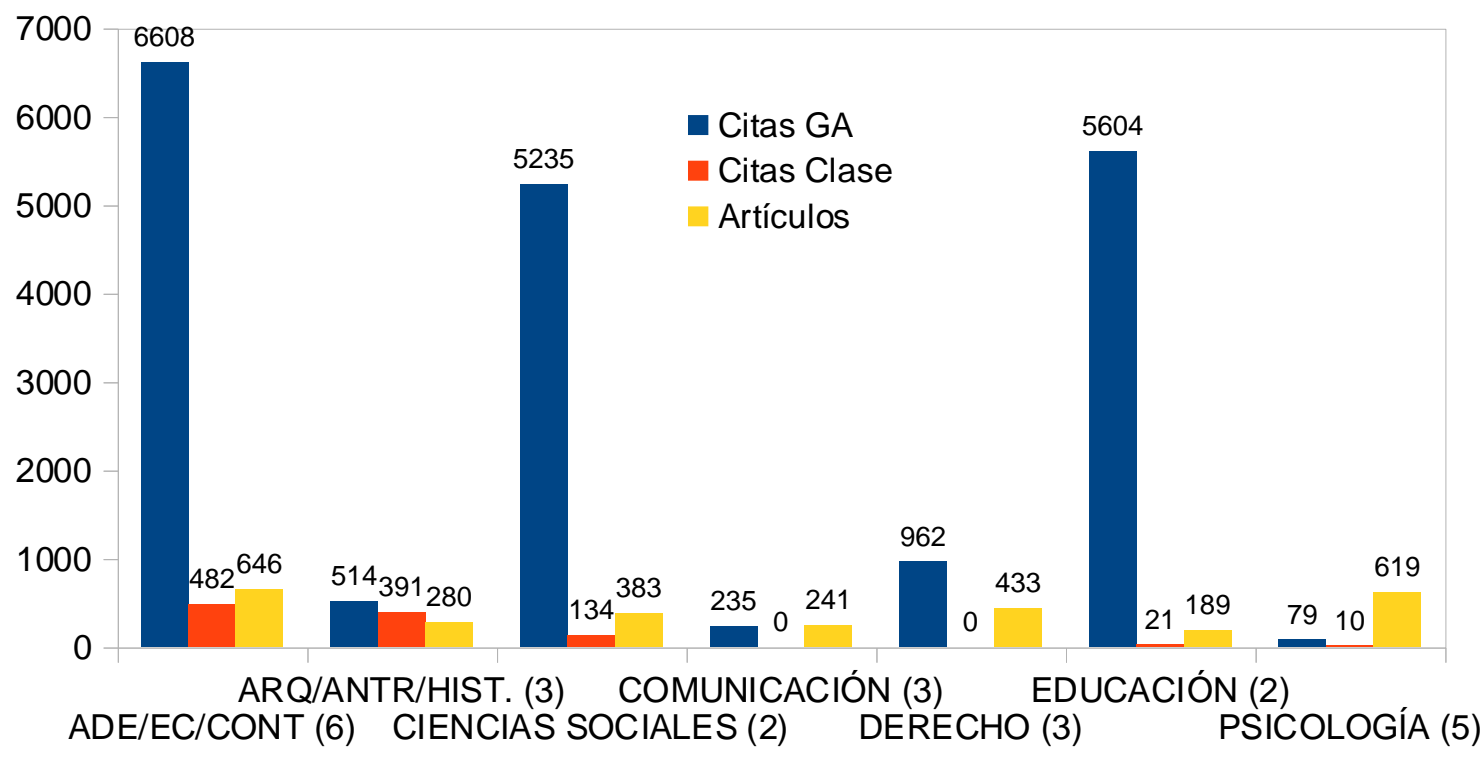


Veamos ahora como se visualiza la correlación de autores por ámbitos para generar nuevas lecturas de los datos (Tabla 11 y Gráfico 7). Observamos que finalmente el área que obtiene el mayor número de autores extranjeros es ARQ/ANT/HIST., con 219 artículos extranjeros y 117 nacionales. Comunicación, que en todas las anteriores tablas estaba relegada a las últimas posiciones, en la vista agrupada mejora sus indicadores con 155 artículos de autores extranjeros y 126 nacionales. El resto de áreas tiene un fuerte predominio de autores nacionales sobre extranjeros. Si bien en el análisis individual algunas revistas de ADE/EC/CONT, mostraban mejor ratio de autoría proveniente del exterior. En la vista global, los números no le resultan tan favorables: 445 nacionales y 391 extranjeros, aún cuando hay que valorar un importante número de artículos en su conjunto: 646. La preferencia hacia los autores peruanos es muy evidente también en áreas como Derecho, Educación y Psicología; donde la cuota nacional es el doble o incluso hasta el triple respecto de los autores provenientes del exterior. En Psicología se da la casuística que habiendo la más elevada producción de literatura, más de 1000 artículos, 638 son de factura nacional. El caso más poderoso sucede en Ciencias Sociales (general) donde en las dos revisas existentes se reportan 423 artículos nacionales y solo 31 de extranjero.

Tabla 11: Tabla comparada de producción por ambitos: Autores

\begin{tabular}{|l|l|c|c|}
\hline & & AN & A. EXT \\
\hline $\mathbf{1}$ & ADE/EC/CONT & 445 & 391 \\
\hline $\mathbf{2}$ & ARQ/ANTR/HIST. & 117 & 219 \\
\hline $\mathbf{3}$ & CIENCIAS SOCIALES & 423 & 31 \\
\hline $\mathbf{4}$ & COMUNICACIÓN & 126 & 155 \\
\hline $\mathbf{5}$ & DERECHO & 335 & 137 \\
\hline $\mathbf{6}$ & EDUCACIÓN & 197 & 56 \\
\hline $\mathbf{7}$ & PSICOLOGÍA & 638 & 437 \\
\hline
\end{tabular}

Gráfico 7: Comparativa de autores nacionales y extranjeros

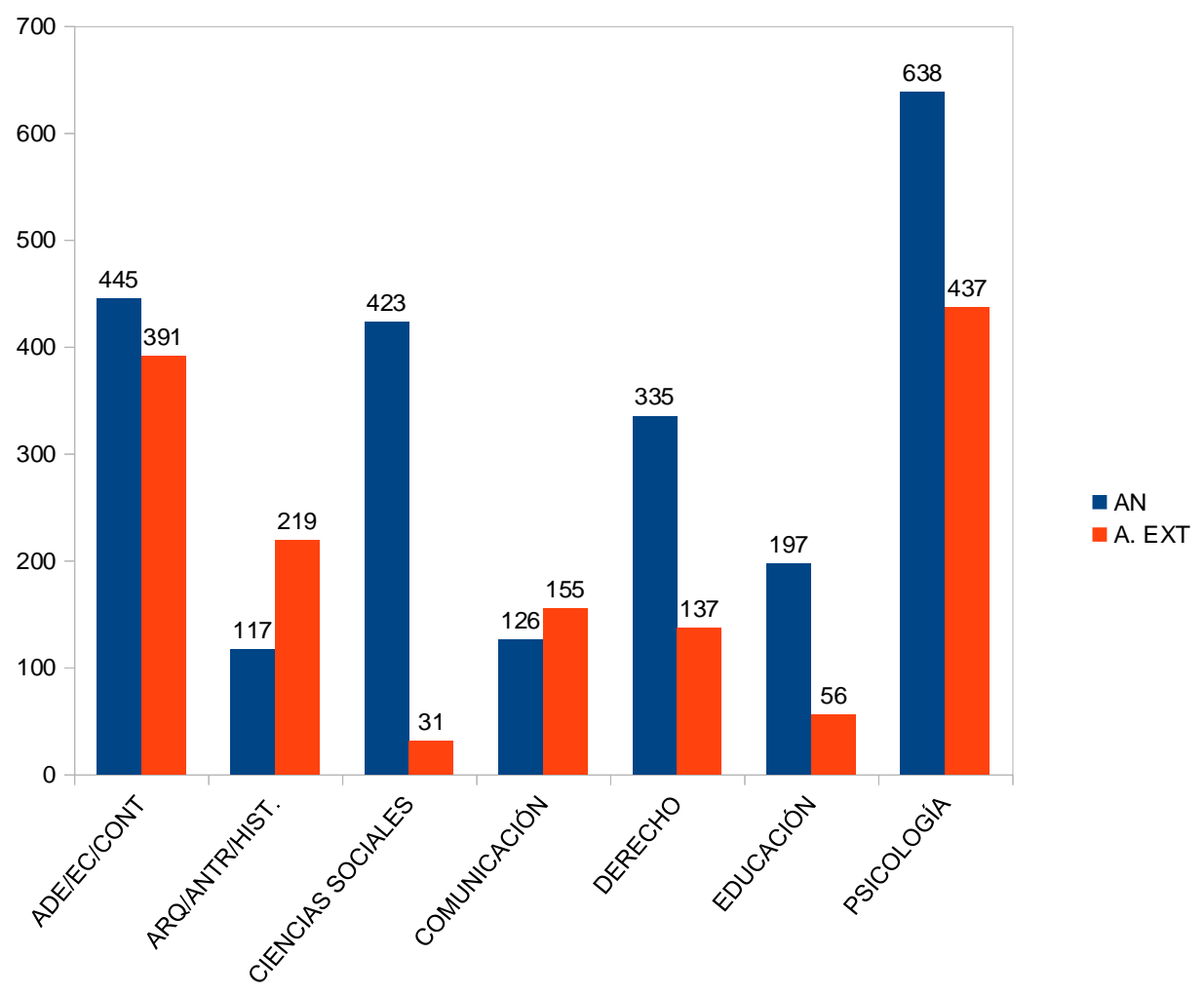

Fuente: El autor (2016). 
Finalmente veamos una tabla (ver Tabla 12) que aglutina los principales indicadores de productividad y visibilidad por universidades.

La PUCP gestiona 10 revistas que alcanzan 1003 artículos. La UNMSM es más productiva, a. alcanza los 767 artículos, pero con solo 4 publicaciones, 6 menos que la PUCP. La combinación de autores nacionales con extranjeros está mucho más equilibrada por la PUCP, una media de 52,28\% de autores extranjeros. Este fenómeno es menos frecuente en las publicaciones de la UNMSM. Como se aprecia en la imagen, los autores nacionales son los que más publican allí y alcanzan el 90,4\%, mientras que los extranjeros representan solo el $9,87 \%$ del total. Las universidades de Lima y Piura, ambas con dos publicaciones cada una, están bastante equilibradas en cuanto a su producción de artículos. Vemos que la Universidad de Piura ha publicado 200, mientras que la Universidad de Lima 198. En cuando se analizan los datos de citaciones las diferencias de las revistas de la Universidad Católica se hacen aún más marcadas respecto del resto de centros. Obtienen en total 3745 citas en Google Académico. En segunda posición encontramos a la Universidad de Piura con 1124, seguida de la Universidad de Lima con 1,073; ambas con 2 revistas. En última posición encontramos a la Universidad de San Marcos solo con 237. Las citas de CLASE son también las más favorables para las revistas de la Universidad Católica, aunque con menor número: 1175. En segundo lugar se encuentra la Universidad de Lima con 849. La Universidad de Piura está mucho más relegada con 99 y cierra la tabla con solo 21 la Universidad de San Marcos.

SciELO reporta 72 citaciones únicamente para revistas de la Universidad Católica. La vista permite comprobar también el número más elevado de visitas a sus portales web $(563,714)$, así como de descargas de artículos $(693,114)$. En segunda posición, bastante emparejados se encuentran la Universidad de Piura y la Universidad de Lima. Observamos que la primera alcanza las 15354 visitas y 13,774 descargas de artículos, mientras que la Universidad de Lima llega a las 13,959 visitas y 10,086 descargas. No hemos podido obtener datos referentes a las 4 publicaciones de la Universidad de San Marcos, que hubiesen resultado muy interesantes para observar el volumen de consultas de sus trabajos y su procedencia. También para indagar acerca de las posibles causas del bajo número de citaciones con tal elevado número de artículos publicados.

Tabla 12: Tabla comparada de productividad entre universidades

\begin{tabular}{|l|c|c|c|c|c|c|c|c|c|}
\hline \multicolumn{1}{|c|}{ Centro } & ReV. & Art. & AN. & A EX & GAC & CLASE & SCIELO & VIS. & DESC. \\
\hline U. Católica & 10 & 1003 & 619 & 742 & 3745 & 1175 & 72 & 563,714 & 693,114 \\
\hline U. San Marcos & 4 & 767 & 1051 & 122 & 237 & 21 & - & - & - \\
\hline U. Piura & 2 & 200 & 194 & 155 & 1,124 & 99 & - & 15,354 & 13,774 \\
\hline U. Lima & 2 & 198 & 112 & 128 & 1,073 & 849 & - & 13,959 & 10,086 \\
\hline
\end{tabular}

En el siguiente gráfico (Gráfico 8) podemos apreciar de forma más nítida el valor de los diferentes indicadores analizados. La barra horizontal más larga indica la amplia ventaja de la Universidad Católica en las citas de GAC, que triplica a las obtenidas por la Universidad de Lima y Universidad de Piura, que superan las 1000. Es incluso superior a la suma de las citas GAC de todas las otras revistas reportadas. En cambio, la barra que grafica las citas de CLASE, podemos encontrara valores bastante igualados entre la Universidad Católica y la Universidad de Lima. Es muy interesante este esquema gráfico, tomando en consideración que la Universidad de Lima lo obtiene solo con dos publicaciones y 198 artículos, frente a 10 revistas y 1003 artículos de la Universidad Católica. 


\section{Gráfico 8: Producción general comparada entre universidades}

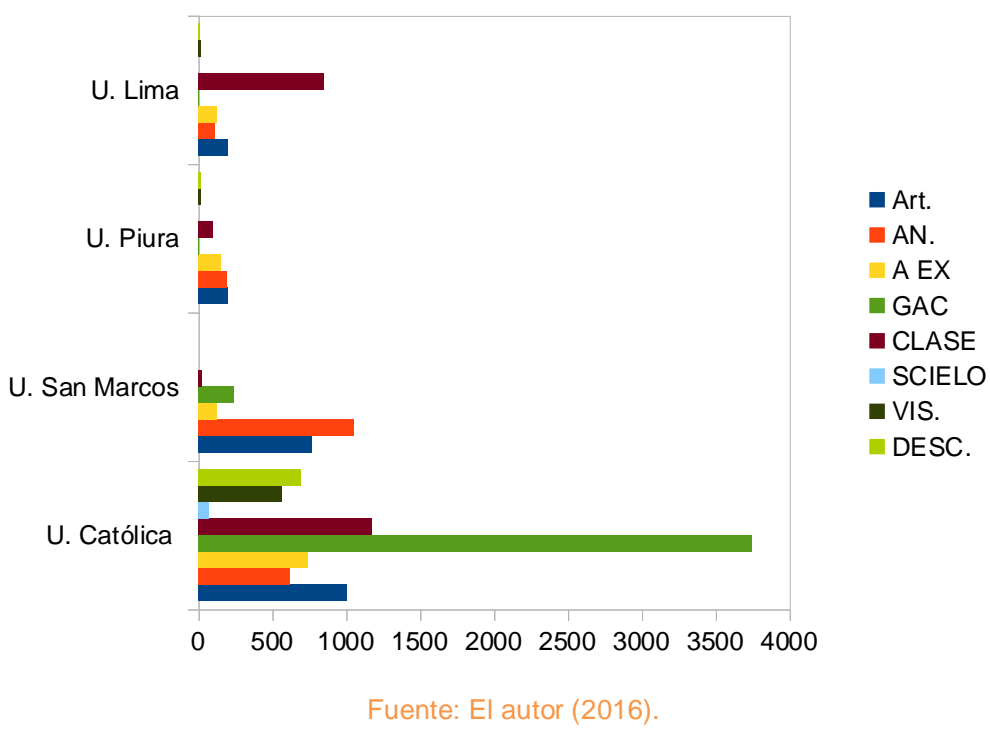

\section{Discusión}

Hemos podido verificar una producción significativa de artículos que alcanza los 2791, distribuidos en 24 publicaciones. Este volumen equivale a una media de 116 artículos por revista. Los resultados permiten observar con claridad que la producción científica en este campo se concentra muy fuertemente en la cuota exhibida por las 10 revistas editadas por la Pontificia Universidad Católica del Perú (Privada) y las 4 de la Universidad Nacional Mayor de San Marcos (Pública). El resto de artículos queda homogéneamente distribuido en otras revistas procedentes de universidades privadas como la Universidad de Piura, Universidad de Lima, Esan y Universidad del Pacífico, así como otras que hace pocos años han comenzado a editar revistas académicas. Mención especial merecen los esfuerzos de la Universidad del Altiplano, universidad pública de la provincia de Puno, cerca de la frontera con Bolivia, por tener una presencia en el conjunto con su revista Comunic@cción.

Se ha podido comprobar que las revistas de la PUCP y UNMSM tienen plataformas propias y completas, dotadas de un sistema de navegación que permite desplazarse con bastante facilidad y obtener los indicadores generales de volúmenes y autores que ofrece por su propia configuración el sistema Open Acces. En cuanto a los números, publican con regularidad, pero muchas de ellas presentan inconvenientes en la gestión de envío de artículos, acceso a revisores temáticos y configuración de perfiles de usuario. En concordancia con los datos obtenidos, se aprecia un trabajo más organizado en la gestión de las publicaciones por parte de la PUCP: selección de autores y variedad temática. Incluso diversifican la procedencia y selección temática de las contribuciones. Hay una mayor preocupación por incluirlas en catálogos como SciELO y CLASE, aunque no todas se encuetran disponibles en ellos. Tienen una buena presencia internacional que se corrobora con un alto número de citaciones. De otra parte, las dos publicaciones que gestionan la Universidad de Lima y la Universidad de Piura consiguen entre sí, resultados bastante similares en cuanto a productividad y citaciones, con una ligera ventaja de la Universidad de Piura, que decididamente otorga mayor preferencia a las contribuciones de autores extranjeros sobre los nacionales. Sus portales están en proceso de implementación del OA y se observa un acceso parcial a sus distintas funciones operativas. En cuanto al volumen de citas obtenidas, en ambas universidades las citas de GAC superan las 1000, pero en CLASE se registra una amplia ventaja de la Universidad de Lima, que alcanza las 849 citaciones sobre las 99 de la Universidad de Piura. Es meritorio que este resultado en la Universidad de Lima se obtenga con una cuota promedio baja de artículos (198). Las 4 revistas de la Universidad de San Marcos, de forma general, dan mucha más preferencia a los autores de la propia universidad sobre los externos nacionales y extranjeros. No obstante, investigan y publican más artículos: 767. Su gran desventaja comparada con el resto de revistas del medio, es que adolecen de visibilidad externa: bajo número de citas y nula presencia en repositorios internacionales. Estas deficiencias han sido advertidas en otros trabajos previos (Huamaní y Pacheco, 2009; Cabezas y Mayta, 2008). Pero no debe 
entenderse, necesariamente, como una falta de interés institucional o mala gestión de sus administradores, sino que podría obedecer a que las temáticas de la mayoría de sus investigaciones son orientadas a problemas nacionales muy acotados, que podrían ser de poco interés para su consulta en el exterior. No hemos podido obtener información de descargas artículos ni de visitas a los portales webs, para tener otros indicios que nos ayuden a explicar su escasa visibilidad internacional. En cualquier caso, a pesar de todo ello, paradójicamente, tienen citas en GAC y algunas otras más en CLASE con su única revista indizada en este catálogo: Investigación Educativa. Hay que señalar, que en todos los casos, la cuantificación de citas de GAC es histórica e incluye todos los artículos publicados desde su primer número. El aporte de las demás revistas queda un tanto eclipsado por el dominio claro de estas universidades. Debemos reconocer un trabajo esmerado de Esan, que tiene un alto número de artículos con autores procedentes de Asia e incluso algunos de Oceanía, fórmula de coautoría poco habitual en revistas nacionales.

El análisis de los autores y su procedencia no es suficiente para poder vincular con precisión instituciones, grupos o centros de investigación, sobre todo externas. Quizás donde se vea mejor algunas conexiones interesantes es en las revistas de economía y arqueología. En ellas el volumen de autores afiliados a centros e instituciones del exterior es bastante elevado y variado en cuanto a sus países de adscripción. Estos datos, de forma indiciaria, reflejan la existencia de redes de contactos institucionales internacionales, a la par de una voluntad por apoyar el trabajo que los ex alumnos están haciendo en el exterior como parte de su formación predoctoral. Para profundizar en este aspecto es necesario tener un registro completo de autores-instituciones, para mesurar el impacto real de los artículos. Actualmente eso no es posible, bien porque las revistas no se encuentran indexadas o porque los datos disponibles de citas son altamente dispares que nos impiden conocer con certeza su diseminación e impacto en los círculos internacionales.

En cuanto al acceso abierto, observamos que es relativo. Los artículos no son de pago y se descargan fácilmente en formato PDF, pero hay algunas revistas que simplemente cargan la versión digital de los números. Esto dificulta el acceso directo a un artículo específico de la búsqueda, la obtención de información de los autores y su centro de adscripción, entre otros indicadores generales. Falta mejorar los protocolos de gestión de usuarios. Las revistas de la PUCP, Universidad de Piura y Universidad de Lima no permiten hacer envíos on line, solo por correo electrónico. La PUCP tampoco deja registrarse como lector ni revisor. Una actitud más abierta y receptiva por parte de los administradores puede mejorar de forma considerable el número, variedad y procedencia tanto de lectores, autores y evaluadores externos. En el mismo sentido, dificulta la búsqueda y descargas artículos para los que acceden desde la plataforma electrónica. Destacamos que solo las revistas de la Universidad de San Marcos operan con todas las funcionalidades disponibles del OA.

\section{Conclusiones y recomendaciones}

Este artículo ha ofrecido los resultados de una investigación bibliométrica de la producción académica en revistas de Ciencias Sociales en el Perú y ha analizado comparadamente algunos indicadores de calidad relevantes. El principal hallazgo ha sido visualizar un claro dominio de dos de las principales universidades peruanas: una privada y otra nacional en la producción de artículos científicos. Existe una cuota importante de autoría externa e internacional, predominantemente en las publicaciones gestionadas por centros privados, que se hacen parcialmente visibles en SciELO, CLASE y Redalyc. Esto es menos frecuente en las publicaciones del resto de instituciones universitarias y nula en el caso de la Revista de Indecopi.

Creemos que el sistema de recolección de datos empleado para esta investigación ha funcionado de manera satisfactoria para analizar el corpus de revistas nacionales de este ámbito. Ha sido una tarea larga y compleja, debido cada uno de los datos se ha introducido de forma manual en el fichero y también porque la información se ha obtenido de numerosas fuentes, no necesariamente conectadas entre sí. Este hecho puede haber provocado algunos errores humanos en el ingreso y cuantificación de los datos por posibles fallos mecánicos. No obstante, ante la inexistencia de una base de datos completa y especializada, representó el único procedimiento posible para construir un mapa actualizado de la producción científica de las revistas peruanas del ámbito de las Ciencias Sociales en el Perú. Debemos asumir también algunas posibles distorsiones en la evolución temporal de las revistas por la falta de volúmenes en algunas de ellas, lo que puede haber afectado en la lectura global y comparada, en especial respecto de otras que sí han publicado con regularidad sus números.

Futuros estudios bibliotecológicos deben orientarse a obtener y examinar la información faltante de algunas variables de métricas. En esta línea, una prioridad es analizar la configuración del mapa de citaciones 
internacionales para conocer con mayor detalle los espacios de proyección de las revistas. Los resultados aquí mostrados son parciales del conjunto generado y resultan del análisis de la totalidad de la información a la cual hemos podido acceder. Aun cuando para este estudio, se hicieron diferentes gestiones para obtenerlos, pudimos constatar poca o nula disposición para bridarlos en algunos casos. La iniciativa de la Universidad Católica de incorporar a varias de sus revistas en SciELO, Redalyc o Clase, debe ser imitada por los demás centros universitarios. Hemos podido ver el efecto positivo de esta decisión en los niveles de lectoría, descargas, citas y factor de impacto. Por otra parte, deben controlarse los procesos editoriales con mayor rigor, cumpliendo con las normas para el recibo de artículos y su gestión a lo largo del proceso de publicación de s revistas. Esto facilita la tarea de la primera revisión por el editor y luego cuando los artículos son enviados a los expertos para el peer review. Deben también respetarse la regularidad de las convocatorias (call for papers) y lanzamiento de nuevos números. Esto influye en la percepción favorable de los colaboradores y la comunidad científica en general. Algunas sospechas de incumplimiento surgen al no haberse detectado correlaciones directas, por ejemplo, entre el número de criterios cumplidos Latindex, con las citas o el factor de impacto suministrado por SciELO. Estas discordancias pueden ser porque indicar los datos ofrecidos son incorrectos o porque no se cumplen con los criterios editoriales debidamente. Otra deficiencia endémica que debe irse reduciendo en el futuro, es la alta tasa endogamia. Los investigadores deben intentar publicar en otras revistas que no son de su propia facultad, no solo para procurar formar nuevas redes de trabajo, sino porque esta mala praxis es penalizada para una futura solicitud de indización en Scopus o ISI de la revista. En ese sentido, hemos detectado algunos casos lamentables de revistas que inclusive no consideran para posible publicación artículos de otras universidades nacionales.

Para trazar un plan hacia el futuro que permita mejores resultados internacionales de las publicaciones científicas, es urgente que el Estado asuma el rol de gran promotor de la investigación nacional. Esta tarea no puede seguir como hasta ahora a la suerte de las iniciativas individuales de cada universidad, porque si no acabará aumentando la brecha entre centros privados y públicos. Las acciones deben enmarcarse como parte de un gran plan nacional del nuevo gobierno, que incluya formación de nuevos doctores, proyectos y recursos destinados a la capacitación investigadora de calidad. Igualmente la formación metodológica y uso eficiente de herramientas estadísticas. De otra parte, ayudas para mejorar la gestión y promoción editorial de revistas científicas. Esto en el futuro revierte positivamente en los centros y docentes, porque abre puertas para trabajos colaborativos en el exterior, participación en proyectos competitivos, etc. Solo en base a una buena gestión de la investigación nacional podrán producirse más y mejores artículos que en un futuro obtengan mejores posiciones y gracias a ellos las revistas podrán constituirse en un núcleo referente para atraer aportaciones de impacto de investigadores internacionales.

\section{Referencias}

Banco Mundial (2015). Perú Panorama General. Recuperado el 1 de marzo de 2016, de: http://www.bancomundial.org/es/country/peru/overview

Borrego, Á.; Urbano, C. (2006). La evaluación de revistas científicas en Ciencias Sociales y Humanidades, Información, cultura y sociedad, n.14, ene./jun. Recuperado el 1 de abril de 2016, de: http://www.scielo.org.ar/scielo.php?script=sci arttext\&pid=S1851-17402006000100002

Burstein, Z.; Cabezas, C. (2010). Advances and challenges of the in the year 2010, Revista Peruana de Medicina Experimental y Salud Pública. Revista Peruana de Medicina Experimental y Salud Pública, 27(1), p. 3-5. Recuperado el 1 de noviembre de 2015, de: http://www.scielosp.org/scielo.php?script=sci arttext\&pid=S1726-46342010000100001

Cabezas, C., \& Mayta-Tristán, P. (2008). Evolución y perspectivas de la Revista Peruana de Medicina Experimental y Salud Pública, 1945-2008. Revista Peruana de Medicina Experimental y Salud Pública, 25(2), 167-168.

Campanario, J. M.; Candelario, A. (2010). La influencia de las autocitas en el aumento del factor de impacto en revistas de Ciencias Sociales. Revista española de documentación científica, 33(2). p. 185-200. Recuperado el 1 de noviembre de 2015, de: http://redc.revistas.csic.es/index.php/redc/article/viewarticle/551

Concytec (2016). Convocatoria Cienciactiva. Recuperado el 1 de marzo de 2016, de:

http://www.cienciactiva.gob.pe/cienciactiva/images/bases/incentivos/bases incentivo_2016.p

Diario Gestión (2014). El número de universidades en el Perú se duplicó en solo 13 años, 5 de julio de 2014. Recuperado el 1 de marzo de 2016, de: http://gestion.pe/economia/numero-universidades-peru-se-duplico-solo-13-anos-2102202

Diario Oficial El Peruano (2014). Ley Universitaria 30220, 9 de julio de 2014. Recuperado el 1 de marzo de 2016, de: http://www.sunedu.gob.pe/nueva-ley-universitaria-30220-2014/ 
Expo Universidad (2014). Situación del sistema universitario peruano, Grupo Educación para el futuro. Recuperado el 1 de marzo de 2016, de: http://educacionalfuturo.com/noticias/regulando-la-calidad-en-la-cantidad-situacion-del-sistemauniversitario-peruano/

Fernández-Quijada, D.; Masip, P.; Bergillos, I. (2013). El precio de la internacionalidad: la dualidad en los patrones de publicación de los investigadores españoles en comunicación. Revista española de documentación científica, 36(2). Recuperado el 1 de octubre de 2015, de: http://redc.revistas.csic.es/index.php/redc/article/viewArticle/792

Fernández-Quijada, D. El perfil de las revistas españolas de comunicación (2007-2008). Revista española de documentación científica, 3(4), p. 553-581. Recuperado el 1 de octubre de 2015, de: http://redc.revistas.csic.es/index.php/redc/article/viewArticle/671

Huamaní, C.; Pacheco-Romero, J. (2009). Visibilidad y producción de las revistas biomédicas peruanas. Revista de Gastroenterología del Perú, 29(2), p. 132-139. Recuperado el 1 de diciembre de 2015, de: http://www.scielo.org.pe/scielo.php?script=sci arttext\&pid=S1022-51292009000200005

Ideas Repec. Recuperado el 1 de junio de 2016, de: https://ideas.repec.org/s/ris/joefas.HTML

Laakso, M.; Welling, P.; Bukvova, H.; Nyman, L.; Björk, B. C.; Hedlund, T. (2011). "The Development of Open Access Journal Publishing from 1993 to 2009". Hermes-Lima, Marcelo. ed.PLoS ONE 6 (6):e20961. Recuperado el 1 de octubre de 2014, de: http://iournals. plos.org/plosone/article?id=10.1371/iournal. pone.0020961

LogEc RePEc (Research Papers in Economics). Recuperado el 1 de junio de 2016, de: http://logec.repec.org/

Miyahira, J. (2011). ¿Más revistas científicas o repositorios institucionales de acceso abierto? Revista Médica Herediana. vol.22, n.1, pp. 1-3. Recuperado el 1 de octubre de 2015, de: http://www.scielo.org.pe/scielo.php?pid=S1018130X2011000100001\&script=sci arttext

Morales, F. (2015). Producción e impacto de las revistas peruanas del ámbito de las Ciencias Sociales en el catálogo Latíndex, Investigación Bibliotecológica, Universidad Autónoma de México, México. In press.

Román Román, A., \& Giménez Toledo, E. (2000). Las revistas españolas de Economía: una propuesta de evaluación científica. Información Comercial Española, ICE: Revista de economía, № 783, 2000, pp. 133-162

Romaní, F.; Wong, P. (2009). Reporte y serie de casos en el Perú: situación de un tipo de publicación subestimada. Análisis de las revistas médicas peruanas indizadas en SciELO-Perú, 1997-2008. Revista peruana de epidemiología 13(3). Recuperado el 1 de noviembre de 2015, de: http://sisbib.unmsm.edu.pe/BVRevistas/epidemiologia/v13 n3/pdf/a09v13n3.pdf

Shintaku, M.; Fagundes, R.; Carvalho, S. (2014). A avaliação dos portais de revistas brasileiros implementados com o seer/ojs por meio do levantamento da indexação pelo Latindex e Scielo, Informaçao \& Sociedade: Est., João Pessoa, v.24, n.2, p. 139148, maio/ago. Recuperado el 1 de enero de 2016, de:http:/www.ies.ufpb.br/ojs2/index.php/ies/article/view/18671/11498

Tavares De Matos Cardoso, M. (2011). El peer review de las revistas científicas en Humanidades y Ciencias Sociales: políticas y prácticas editoriales declaradas. Revista española de documentación científica, 34(2). Recuperado el 1 de noviembre de 2014, de: http://redc.revistas.csic.es/index.php/redc/article/viewArticle/690

Torres-Salinas, D., Delgado López-Cózar, E., \& Jiménez-Contreras, E. (2009). Citation networks among Spanish Socia Sciences journals 1994-2006. Revista Española de Documentación Científica, 32(2), 34-50.

Universidad Continental, Acreditación Internacional Aneca. Recuperado el 1 de marzo de 2016, de: http://www.universidad.continental.edu.pe/acreditaciones/

Universidad de San Martin de Porres, Facultad de Derecho, Acreditacón Internacional AQ. Recuperado el 1 de marzo de 2016, de: http://www.derecho.usmp.edu.pe/acreditacion internacional2015.html

Universidad de Ciencias Aplicadas, Acreditacion Claep. Recuperado el 1 de marzo de 2016, de: http://blogs.upc.edu. pe/noticias-pregrado/facultades-y-alumnos/carrera-de-comunicacion-y-periodismo-de-la-upc-logra-su 


\section{Datos del autor}

\section{Luís Fernando Morales Morante}

Doctor en Comunicación Audiovisual y Publicidad por la Universidad Autónoma de Barcelona (UAB). Diploma de estudios avanzados (DEA) en Suficiencia Investigadora (UAB). Máster en Inicio a la Investigación en Comunicación Audiovisual y Publicidad (UAB). Licenciatura en Ciencias de la Comunicación por la Universidad de Lima.

fernando.morales@uab.es

Recibido - Received: 2016-04-29

Aceitado - Accepted: 2016-06-26

\footnotetext{
i. Se escoge dicho período porque marca un intervalo amplio, suficiente para poder advertir la evolución de las publicaciones y obtener, finalmente, una perspectiva longitudinal de la investigación reciente en este ámbito de conocimiento.

ii. Se opta por este catálogo debido a la inexistencia de un catálogo nacional y porque en Latindex Catálogo hay un volumen apreciable de revistas de Ciencias Sociales incluidas, que permiten bosquejar un mapa completo de la literatura en sus diferentes subareas temáticas.

iii. Revista de Derecho de la Pontificia Universidad Catolica del Perú

iv. Revista de Psicologia de la Pontificia Universidad Catolica del Perú

v. Revista de Psicologia de la Universidad Cesar Vallejo

vi. Agradecemos a las personas responsables de los portales de las revistas que gentilmente nos han cedido la información para confeccionar esta tabla: Ismael Canales, Yuri Rosas: Pontificia Universidad Católica del Perú, Rosa Zeta Pozo y Noemí Medina: Universidad de Piura, Nelly Casas y Dennis Cuéllar: Universidad de Lima, Jorge Guillén (Journal of Economics).

vii. Fuente PUCP

viii. Fuente Redalyc

ix. Fuente Redalyc
}

This work is licensed under a Creative Commons Attribution 4.0

United States License.

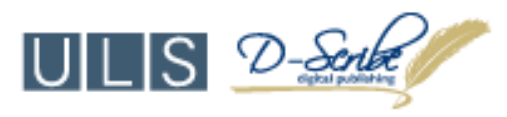

This journal is published by the University Library System of the University of Pittsburgh as part of its D-Scribe Digital Publishing Program and is cosponsored by the University of Pittsburgh Press. 